\begin{abstract}
In longitudinal models with time-varying predictors, the need to distinguish their within-person (WP) relations of time-specific residuals from their between-person (BP) relations of individual means is relatively well-known. In contrast, the need to further distinguish their BP relations of individual time slopes has received much less attention. This article addresses the deleterious impact that ignoring effects of individual time slopes in time-varying predictors can have on the recovery of BP intercept and WP residual relations in commonly used variants of longitudinal models. Using simulation methods and analyses of example data, this problem is demonstrated within univariate longitudinal models (i.e., multilevel or mixed-effects models using observed predictors), as well as in multivariate longitudinal models (i.e., structural equation models using latent predictors, including those for cross-lagged relations). Recommendations are provided for how to avoid conflating the BP and WP associations of longitudinal variables in practice.
\end{abstract}

Key Words: time-varying predictors, multilevel models, structural equation models, conflated effects, smushed effects, cross-lagged panel models 


\section{Disaggregating Associations of Between-Person Differences in Change over Time from Associations of Within-Person Fluctuation in Longitudinal Data}

Longitudinal designs create great opportunities for examining across-variable relations at multiple levels of analysis, with a wide variety across studies in how often people are sampled. For instance, in studying the effects of health on long-term changes in cognition, persons might be sampled once a year, whereas in studying the effect of health on short-term fluctuations in mood, persons might be sampled once a day. Although the goal of longitudinal sampling is to capture within-person changes over time, any variable measured repeatedly also usually contains systematic between-person differences, such as in its mean level over time. Consequently, in examining time-varying predictors of time-varying outcomes, one must be careful to distinguish different forms of between-person (BP) and within-person (WP) relationships. As an example of a BP relation, persons with higher average levels of a predictor over time (relative to other people) may also have higher average levels of an outcome over time (relative to other people). WP relations can then occur through time-specific fluctuations around these person means. For example, when a predictor is higher than usual (relative to a person's average), an outcome may also be higher than usual, either for an outcome measured at the same occasion as the predictor (a concurrent relation), or for an outcome measured at a subsequent occasion (a lagged relation).

Given the prominent role of time in longitudinal studies, time-varying predictors and outcomes may be associated in other ways as well. In particular, the previous BP example refers only to an association of individual mean differences across variables, or an intercept relation. But individual differences related to change over time may also be present in any variable measured repeatedly, which creates an opportunity for BP associations of these individual time slopes as well, particularly in longer-term studies. As an example, while people who are healthier 
on average (than other people) may have better cognitive functioning on average (than other people; a BP intercept relation), people who decline less in health over time (than other people) may also decline less in cognition over time (than other people). The latter is also a BP relation, but among individually-varying time slopes instead of individual intercepts.

Time slope associations may be relevant in studies of shorter-term fluctuation as well, albeit in a modified form. For instance, persons with fewer daily health problems on average (than other people) may report better daily moods on average (than other people; a BP intercept relation). Although systemic change in either variable may be unlikely over the course of a few weeks, short-term trends (such as weekly cycles) may still create individual differences related to effects of time. For example, people may differ in the extent to which they report more problems on Mondays than on other days (a "Monday effect"). But if people who exhibit a larger Monday effect in their health problems also exhibit a larger Monday effect in their moods, the relation of these individual "Monday" slopes creates a distinct BP association between health and mood.

\section{The Current Study}

Unfortunately, failing to address potential BP relations among time-varying predictors and outcomes of their individual time slopes (in addition to their BP intercept relations) can result in incorrect inferences for their WP associations, which become confounded with the unmodeled time slope relation. Although the need to distinguish BP relations among individual mean differences when assessing WP relations is well-known (e.g., Algina \& Swaminathan, 2001; Berry \& Willoughby, 2017; Curran et al., 2012; Hamaker et al., 2015; Hoffman, 2015, ch. 8; Hoffman \& Stawski, 2009), less well-understood is the deleterious impact of unmodeled BP time slope relations. Previous work concerning the role of time in assessing relations across longitudinal variables has been more fragmented - by referring to a single modeling framework 
(e.g., univariate multilevel models in Curran \& Bauer, 2011, and Wang \& Maxwell, 2015; or structural equation models in Curran et al., 2014, and Scott, 2020), or by addressing only one type of WP association (e.g., concurrent in Hoffman, 2015, ch. 9; or lagged in Scott, 2020).

Accordingly, the purpose of the current study is two-fold: (1) to raise awareness of the confounding role of BP relations of individual time slopes in assessing concurrent or crosslagged WP relations, and (2): to demonstrate how this confounding can or cannot be avoided across common variants of univariate and multivariate longitudinal models. To help readers better understand their similarities and differences, each model that follows is described using both multilevel model (MLM) equations and single-level structural equation model (SEM) diagrams. Their specification as multilevel SEMs is also described in the online supplement.

The remainder of this article is organized as follows. First is a review of unconditional models for change over time and concerns about levels of analysis for time-varying predictors. Next is a review of univariate longitudinal models that can distinguish BP intercept from WP residual associations (using person-mean-centering or baseline-centering for time-varying predictors), followed by multivariate longitudinal models that can do so (using latent centering for time-varying predictors), in which cross-lagged (bidirectional) WP residual associations can also be examined. Then described is the confounding role of individual change over time in a time-varying predictor in assessing its BP intercept and WP residual relations. The extent to which each model variant can mitigate these problems is then demonstrated using simulation methods and analyses of motivating example data. Lastly, recommendations are given on how to avoid an incomplete disaggregation of BP relations for individual time slopes in practice.

A motivating example is provided by results from Robitaille et al. (2013), who examined longitudinal mediation of age changes in memory (measured by prose recall) from age changes 
in processing speed (measured by digit-symbol substitution) in 470 older adults (same-sex twins) assessed every two years for up to five occasions. These results were used to simulate data by which to illustrate the models presented below. Relative to the original study, the simulated data were simplified — to reflect independent persons, no baseline age variance, and no attrition — in order to focus solely on disentangling BP from WP associations among longitudinal variables.

\section{An Unconditional Univariate Longitudinal Model for Change over Time}

Each longitudinal model to be examined has a common starting point — an unconditional model for change over time (i.e., with no predictors besides time). A univariate longitudinal model predicting change in memory across five occasions is shown in Equation 1 below:

WP Level-1 Model and Matrix $\boldsymbol{R}$ : $\quad$ BP Level-2 Model and Matrix $\boldsymbol{G}$ :

$$
\begin{aligned}
& y_{t i}=\beta_{0 i}+\beta_{1 i}\left(\text { Time }_{t i}\right)+e_{t i} \quad \text { Intercept: } \quad \beta_{0 i}=\gamma_{00}+U_{0 i} \\
& \boldsymbol{R}:\left[\begin{array}{l}
e_{0 i} \\
e_{1 i} \\
e_{2 i} \\
e_{3 i} \\
e_{4 i}
\end{array}\right] \sim \operatorname{MVN}\left[\begin{array}{l}
0 \\
0 \\
0 \\
0 \\
0
\end{array}\right],\left[\begin{array}{ccccc}
\sigma_{e}^{2} & 0 & 0 & 0 & 0 \\
0 & \sigma_{e}^{2} & 0 & 0 & 0 \\
0 & 0 & \sigma_{e}^{2} & 0 & 0 \\
0 & 0 & 0 & \sigma_{e}^{2} & 0 \\
0 & 0 & 0 & 0 & \sigma_{e}^{2}
\end{array}\right] \quad \boldsymbol{G}:\left[\begin{array}{l}
U_{0 i} \\
U_{1 i}
\end{array}\right] \sim \mathrm{MVN}\left[\begin{array}{l}
0 \\
0
\end{array}\right],\left[\begin{array}{cc}
\tau_{U_{0}}^{2} & \tau_{U_{0}, U_{1}} \\
\tau_{U_{0}, U_{1}} & \tau_{U_{1}}^{2}
\end{array}\right]
\end{aligned}
$$

using multilevel model (MLM) notation over two levels: occasions at level 1 (as shown on the left), and persons at level 2 (as shown on the right). This two-level model requires a long (or stacked) data structure, with one row per occasion per person (and one column per variable).

In the level-1 model, $y_{t i}$ is the memory outcome, and the residual $e_{t i}$ is the difference between the predicted and observed outcome at time $t$ for person $i$. The level-1 equation for $y_{t i}$ contains an individual intercept $\beta_{0 i}$ and an individual slope $\beta_{1 i}$ for linear Time ti $_{\text {(coded } 0-4 \text { to }}$ represent time-since-baseline as equidistant intervals). In the level-2 model, each $\beta_{i}$ is defined by its own equation including a fixed effect ( $\gamma_{00}$ for the intercept, $\gamma_{10}$ for the time slope), which is the average effect for the sample, and a random effect ( $U_{0 i}$ for the intercept, $U_{1 i}$ for the time slope), which is the deviation from the fixed effect for person $i$. The level-2 matrix $\boldsymbol{G}$ holds the 
variances and covariances of the $U_{i}$ random effects - these are the BP differences to be explained by level-2 time-invariant predictors. The level-1 matrix $\boldsymbol{R}$ holds the variances and covariances of the $e_{t i}$ per-occasion residuals - these WP differences that remain after controlling for individual change over time can in turn be explained by level-1 time-varying (longitudinal) predictors. Although shown for five occasions here, $\boldsymbol{R}$ can be generalized to any number of occasions. - Insert Figure 1 about here -

Another way of estimating Equation 1 is a single-level structural equation model (SEM), as shown in Figure 1, which instead requires a multivariate or wide data structure with one row per person (and one column per variable per occasion). The five occasions (observed outcomes in rectangles with time-specific labels) are predicted by two latent factors (in ovals) that covary (via an undirected arrow). The meaning of these latent factors is set by constraining their factor loadings (directed arrows from the ovals to the rectangles): to 1 (to create a latent intercept, $\beta_{0 i}$ ) or to the Time $e_{t i}$ value for that occasion (to create a latent linear time slope, $\beta_{1 i}$ ). The latent factor means are the fixed effects $\gamma_{00}$ and $\gamma_{10}$, and the factor variances and covariances are those of the BP random effects $\left(U_{0 i}\right.$ and $\left.U_{1 i}\right)$ in the level-2 matrix $\boldsymbol{G}$. The intercepts of the five occasion variables are fixed to zero, and their residual variances - the WP variances of the $e_{t i}$ residuals in the level-1 matrix $\boldsymbol{R}$ - are held equal (as shown by the shared rounded rectangle, a convention used here to reduce clutter relative to the inclusion of five separate residual ovals).

Before adding time-varying predictors, we need to acknowledge seven assumptions that underlie this model and those that follow. (1) The isomorphism of the MLM equation and SEM diagram is afforded by a design with balanced time; otherwise, definition variables would be needed to replace the fixed factor loadings for the time slope $\beta_{1 i}$ with individually-varying factor loadings to maintain equivalent results. (2) A random linear time slope adequately captures the 
average trajectory of change over time and individual differences therein; otherwise, additional fixed and random effects for nonlinear change (or estimated loadings for the time slope factor) can be included. (3) The level-2 random effects and the level-1 residuals both follow multivariate normal distributions, but other level-1 conditional distributions can be used instead. (4) The person random effects are independent; otherwise, additional levels of random effects to predict correlations between persons can be included. (5) All heterogeneity of variance and covariance over time has been captured by the level-2 random effects, such that the level-1 residuals are independent with equal variance across time (as shown in the level-1 matrix $\boldsymbol{R}$ ). Otherwise, residual covariances (and/or different variances by occasion) can be specified. (6) No occasionspecific effects are present within the level-1 residuals; otherwise, such stochastic impulses could be considered as well. (7) Most relevant to the point of this paper, in this example the level-1 Time $_{t i}$ predictor has no individual mean variation - in practice this condition will be violated whenever people begin the study at different values of the predictor capturing the passage of time (e.g., age, grade in school, or time since an event; for solutions, see Hoffman, 2012).

\section{Understanding Levels of Analysis for Time-Varying Predictors}

Building on Equation 1, a naïve attempt to examine the effect on memory of a new $x_{t i}$ time-varying predictor (speed) might look like the conditional model in Equation 2 below:

$$
\begin{array}{ll}
\text { WP Level-1 Model: } & \text { BP Level-2 Model: } \\
y_{t i}=\beta_{0 i}+\beta_{1 i}\left(\text { Time }_{t i}\right) & \text { Intercept: } \quad \beta_{0 i}=\gamma_{00}+U_{0 i} \\
+\beta_{2 i}\left(x_{t i}\right)+e_{t i} & \text { Time Slope: } \beta_{1 i}=\gamma_{10}+U_{1 i} \\
& \text { WP } x_{t i}^{*} \text { Slope: } \beta_{2 i}=\gamma_{20}
\end{array}
$$

in which the level-1 matrix $\boldsymbol{R}$ and level-2 matrix $\boldsymbol{G}$ remain the same as in Equation 1. A new $\beta_{2 i}$ individual slope for $x_{t i}$ (grand-mean-centered time-varying speed) has been added to the level-1 model, which is defined by only a fixed effect $\gamma_{20}$ in the level-2 model. (The choice to omit an individual random slope $U_{2 i}$ for the level-1 effect of speed predicting memory - made only to 
simplify the more complex models that follow-will be revisited in the discussion.)

— Insert Figure 2 about here -

Although it may seem natural to treat time-varying speed similarly as time (that has only WP variation here), Equation 2 will likely yield incorrect results for any time-varying predictor that contains BP variation beyond its expected WP variation. Its proportion of BP intercept variation can be indexed using an intraclass correlation (ICC) from an empty means, random intercept model (i.e., without random effects of time) predicting the time-varying predictor itself. An ICC $>0$ indicates that people differ in their mean levels, such that the time-varying predictor is really (at least) two variables instead of one - a BP intercept (person mean differences), and a WP residual (time-specific person-mean deviations). The predictor's BP intercept has a potential level-2 fixed effect (that explains the level-2 random intercept variance $\tau_{U_{0}}^{2}$ ), and its WP residual has a potential level-1 fixed effect (that explains the level-1 residual variance $\sigma_{e}^{2}$ ).

Without separating the two variables contained in a time-varying predictor, its fixed effect $\gamma_{20}$ will be a useless blend of its BP intercept and WP residual effects (weighted towards the WP residual effect; see Raudenbush \& Bryk, 2002, ch. 5). Here, this implies that the effect on average memory of being a person with faster processing speed than others (the BP intercept effect) is the same as the effect on memory at that occasion of being faster than usual (the WP residual effect). This useless blended effect has been called a conflated effect (e.g., Preacher et al., 2011), a convergence effect (e.g., Sliwinski et al., 2010), a composite effect (e.g., Wang \& Maxwell, 2015), and a smushed effect (e.g., Hoffman, 2015); the latter term will be used here. A corresponding SEM diagram for Equation 2 is shown in Figure 2, in which the paths from each $x_{t i}$ observed predictor to the $y_{t i}$ outcome at the same occasion (a concurrent relation) share a common $\gamma_{20}$ fixed effect. The long-dashed arrows from the $x_{t i}$ observed predictors to the 
level-2 intercept factor are not formally included in the model - they are used to schematically illustrate that fact that $\gamma_{20}$ also represents the BP intercept effect. As discussed elsewhere (e.g., Curran et al., 2012), although estimating covariances of the level-2 intercept factor with the timevarying predictors results in the fixed effect $\gamma_{20}$ becoming a pure WP residual effect as intended, this strategy then does not allow a predictive BP intercept effect to be included in the model.

Significant attention has been given to the problem of smushed effects and how to modify the model in Equation 2 to represent distinct BP intercept and WP residual fixed effects of a time-varying predictor. The two main strategies for doing so-within univariate or multivariate longitudinal modeling — are described next, in which the primary difference lies in how the timevarying predictor is represented (using observed or latent predictor variables, respectively). The model in Equation 2 (and all models presented in turn below) are also listed in Appendix A, along with a brief description of the potential bias in each fixed effect.

\section{Univariate Longitudinal Models for BP Intercept and WP Residual Associations}

One method by which to distinguish the BP intercept and WP residual effects of an $x_{t i}$ time-varying predictor uses observed predictor variables, as shown in Equation 3 below:

$$
\begin{array}{ll}
\text { WP Level-1 Model: } & \text { BP Level-2 Model: } \\
y_{t i}=\beta_{0 i}+\beta_{1 i}\left(\text { Time }_{t i}\right) & \text { Intercept: } \quad \beta_{0 i}=\gamma_{00}+\gamma_{01}\left(x_{i}^{*}\right)+U_{0 i} \\
+\beta_{2 i}\left(x_{t i}^{*}\right)+e_{t i} & \text { Time Slope: } \beta_{1 i}=\gamma_{10}+\gamma_{11}\left(x_{i}^{*}\right)+U_{1 i} \\
& \text { WP } x_{t i}^{*} \text { Slope: } \beta_{2 i}=\gamma_{20}
\end{array}
$$

in which the level-2 matrix $\boldsymbol{G}$ and level-1 matrix $\boldsymbol{R}$ are as given in Equation 1, and two new observed predictor variables have been added: level-1 $x_{t i}^{*}$ and level- $2 x_{i}^{*}$. For each $\gamma$ fixed effect or $U$ random effect, the first subscript indexes the level-1 $\beta$ to which it belongs. For the fixed effects, the second subscript indexes its order in the level-2 equation. For the random effects, the second subscript indicates the sampling unit it varies over ( $i$ for persons here). A corresponding SEM diagram is shown in Figure 3a, in which a new observed predictor rectangle for level-2 $x_{i}^{*}$ 
has been added. We will consider two univariate models with this format, as described next.

— Insert Figure 3 about here -

\section{Person-Mean-Centering for Time-Varying Predictors}

One variant uses a strategy called person-mean-centering (PMC; Algina \& Swaminathan, 2001; Curran \& Bauer, 2011; Hoffman, 2015, ch. 8; Hoffman \& Stawski, 2009; Wang \& Maxwell, 2015), in which a new level-1 $x_{t i}^{*}$ predictor is created by centering time-varying $x_{t i}$ with a variable for each person's mean: level-1 $x_{t i}^{*}=x_{t i}-\bar{x}_{i}$. Under the belief that level-1 $x_{t i}^{*}$ should then contain only WP variation, its fixed effect $\gamma_{20}$ should become the WP residual effect as intended. Here, the level-1 fixed effect $\gamma_{20}$ would represent the effect of greater speed than usual on memory at that occasion (after controlling for individual change in memory over time). Although $\gamma_{20}$ is a concurrent WP effect here, a lagged WP effect could also be examined by adding a new version of the $x_{t i}^{*}$ predictor created from $x_{t i}$ at the previous occasion instead.

The level- $2 x_{i}^{*}$ predictor is then formed by centering the $\bar{x}_{i}$ person mean with a constant $C$ (e.g., the grand mean) so that 0 is meaningful: level-2 $x_{i}^{*}=\bar{x}_{i}-C$. In the level-2 equation for $\beta_{0 i}$, the $\gamma_{01}$ fixed effect of level-2 $x_{i}^{*}$ is its BP intercept effect on the $y_{t i}$ intercept - the effect of more $\bar{x}_{i}$ than other people on the predicted $y_{t i}$ at time $=0$. In the level-2 equation for $\beta_{1 i}$, the $\gamma_{11}$ fixed effect of level-2 $x_{i}^{*}$ is its BP intercept effect on the $y_{t i}$ time slope - the effect of more $\bar{x}_{i}$ than other people on the change in $y_{t i}$ over time (also known as a cross-level interaction). Here, these fixed effects would indicate to what extent persons with faster average processing speed (than others) differ in their initial memory $\left(\gamma_{01}\right)$ and in their change in memory over time $\left(\gamma_{11}\right)$.

\section{Baseline-Centering for Time-Varying Predictors}

An advantage of PMC is its use of all occasions to remove time-specific variance from the level-2 $x_{i}^{*}$ predictor, but this can result in a disadvantage for its interpretation. When time is 
centered so that baseline $=0$ (a frequent choice, as made here), PMC creates an asynchrony in the level-2 variables: Whereas the level-2 $x_{i}^{*}$ predictor reflects average BP differences over time, the level-2 intercept $\beta_{0 i}$ reflects BP differences in $y_{t i}$ at time $=0$. Here, using PMC would tell us how BP differences in speed at the mean occasion predict BP differences in initial memory and change in memory over time. One could resolve this asynchrony by also mean-centering time, but if BP differences at baseline are of more interest, an alternate strategy may be considered.

In baseline-centering (BLC; Hoffman, 2012; Sliwinski \& Buschke, 2004), the $\bar{x}_{i}$ person mean is replaced by $x 0_{i}, x_{t i}$ at time $=0$, as follows: level-1 $x_{t i}^{*}=x_{t i}-x 0_{i}$; level-2 $x_{i}^{*}=x 0_{i}-$ $C$. Using BLC, the effects of level-2 $x_{i}^{*}$ on the $\beta_{0 i}$ intercept $\left(\gamma_{01}\right)$ and $\beta_{1 i}$ time slope $\left(\gamma_{11}\right)$ then tell us how BP differences in initial speed predict BP differences in initial memory and change in memory over time. The $\gamma_{20}$ fixed effect of level- $1 x_{t i}^{*}$ should provide the WP residual effect of more speed than at baseline on memory at that occasion (after controlling for individual change in memory over time). A disadvantage of using BLC is that the observed $x_{t i}$ at time $=0$ will also contain WP residual variance as well the intended BP intercept (baseline) variance.

\section{Multivariate Longitudinal Models for BP Intercept and WP Residual Associations}

\section{Concurrent WP Residual Associations}

An alternative strategy for distinguishing the BP intercept and WP residual effects of time-varying predictors also addresses the asymmetry in how $x_{t i}$ and $y_{t i}$ are treated. Why should we try to partition sources of BP and WP variance using observed variables for time-varying predictors, but do so using model- estimated variance components for time-varying outcomes? The capacity to treat both level-1 variables similarly — using latent variables — is the primary benefit of multivariate longitudinal models. To illustrate, the univariate longitudinal model in Equation 3 predicting $y_{t i}$ (memory only) has been transformed into a multivariate longitudinal 
model predicting both $x_{t i}$ and $y_{t i}$ (speed and memory simultaneously) in Equation 4 below:

WP Level-1 Model and Level-1 matrix $\boldsymbol{R}$ :

$$
\begin{aligned}
& x_{t i x}=\beta_{0 i x}+x w_{t i x} \\
& x w_{t i x}=e_{t i x} \\
& y_{t i y}=\beta_{0 i y}+\beta_{1 i y}\left(\text { Time }_{t i}\right)+y w_{t i y} \\
& y w_{t i y}=\beta_{2 i y}\left(x w_{t i x}\right)+e_{t i y} \\
& \boldsymbol{R}:\left[\begin{array}{l}
e_{0 i} \\
e_{1 i} \\
e_{2 i} \\
e_{3 i x} \\
e_{4 i x} \\
e_{0 i y} \\
e_{1 i y} \\
e_{2 i} \\
e_{3 i y} \\
e_{4 i y}
\end{array}\right] \sim \mathrm{MVN}\left[\begin{array}{l}
0 \\
0 \\
0 \\
0 \\
0 \\
0 \\
0 \\
0 \\
0 \\
0
\end{array}\right],\left[\begin{array}{cccccccccc}
\sigma_{e_{x}}^{2} & 0 & 0 & 0 & 0 & - & 0 & 0 & 0 & 0 \\
0 & \sigma_{e_{x}}^{2} & 0 & 0 & 0 & 0 & - & 0 & 0 & 0 \\
0 & 0 & \sigma_{e_{x}}^{2} & 0 & 0 & 0 & 0 & - & 0 & 0 \\
0 & 0 & 0 & \sigma_{e_{x}}^{2} & 0 & 0 & 0 & 0 & - & 0 \\
0 & 0 & 0 & 0 & \sigma_{e_{x}}^{2} & 0 & 0 & 0 & 0 & - \\
- & 0 & 0 & 0 & 0 & \sigma_{e_{y}}^{2} & 0 & 0 & 0 & 0 \\
0 & - & 0 & 0 & 0 & 0 & \sigma_{e_{y}}^{2} & 0 & 0 & 0 \\
0 & 0 & - & 0 & 0 & 0 & 0 & \sigma_{e_{y}}^{2} & 0 & 0 \\
0 & 0 & 0 & - & 0 & 0 & 0 & 0 & \sigma_{e_{y}}^{2} & 0 \\
0 & 0 & 0 & 0 & - & 0 & 0 & 0 & 0 & \sigma_{e_{y}}^{2}
\end{array}\right]
\end{aligned}
$$

BP Level-2 Model and Level-2 Matrix $\boldsymbol{G}$ :

$$
\begin{array}{ll}
x_{t i x} \text { Intercept: } & \beta_{0 i x}=\gamma_{00 x}+U_{0 i x} \\
y_{t i y} \text { Intercept: } & \beta_{0 i y}=\gamma_{00}+\gamma_{01 y}\left(\beta_{0 i x}\right)+U_{0 i y} \\
y_{t i y} \text { Time Slope: } \quad \beta_{1 i y}=\gamma_{10 y}+\gamma_{11}\left(\beta_{0 i x}\right)+U_{1 i y} \\
y w_{t i y} \text { WP } x_{t i x}^{*} \text { Slope: } \beta_{2 i y}=\gamma_{20 y} \\
\text { G: }\left[\begin{array}{l}
U_{0 i x} \\
U_{0 i y} \\
U_{1 i y}
\end{array}\right] \sim \operatorname{MVN}\left[\begin{array}{l}
0 \\
0 \\
0
\end{array}\right],\left[\begin{array}{ccc}
\tau_{U_{0 x}}^{2} & - & - \\
- & \tau_{U_{0 y}}^{2} & \tau_{U_{0 y}, U_{1 y}} \\
- & \tau_{U_{0 y}, U_{1 y}} & \tau_{U_{1 y}}^{2}
\end{array}\right]
\end{array}
$$

in which a third subscript has been added to denote which variable is referred to by each term.

At level 2, $y_{\text {tiy }}$ (memory) is still predicted by the intercept $\beta_{0 i y}$ and time slope $\beta_{1 i y}$, although to parallel Equation 3, $x_{\text {tix }}$ (speed) is predicted only by an intercept $\beta_{0 i x}$. At level 1, the $x w_{\text {tix }}$ and $y w_{\text {tiy }}$ terms capture the WP variation that remains after controlling for individual BP differences in each variable. The $\tau_{U_{0 x}}^{2}$ variance of the speed intercept $\beta_{0 i x}$ is now included in the level-2 matrix $\boldsymbol{G}$, and the level-1 matrix $\boldsymbol{R}$ has been expanded to include the time-specific $x_{\text {tix }}$ residuals (with equal variance $\sigma_{e_{x}}^{2}$ and no covariances over time). In each matrix, dashes indicate 
across-variable covariances that cannot be estimated because those relations are represented by fixed effects instead. These include $\gamma_{01 y}$ and $\gamma_{11}$, the fixed effects of the speed intercept $\beta_{0 i x}$ (which replaced the observed level-2 predictor $x_{i}^{*}$ ) in predicting the memory intercept $\beta_{0 i y}$ and time slope $\beta_{1 i y}$, as well as $\gamma_{20 y}$, the fixed effect of the speed residual $x w_{t i x}$ (which replaced the observed level-1 predictor $x_{t i}^{*}$ ) in predicting the memory WP term $y w_{t i y}$. Figure $3 \mathrm{~b}$ provides a corresponding SEM diagram - the first variant that can be considered a multivariate longitudinal model - in which latent variables now predict both $x_{t i x}$ and $y_{t i y}$, and the latent variables for $x_{t i x}$ (speed) in turn predict the latent variables for $y_{t i y}$ (memory). This is in contrast to the univariate longitudinal SEM shown in Figure 3a (so-named given the use of observed $x_{t i}$ variables in predicting the latent $y_{t i y}$ variables, which means that $y_{t i y}$ is the only outcome being predicted). This multivariate longitudinal strategy (of treating time-varying predictors just like timevarying outcomes) is also known as latent centering (e.g., Asparouhov \& Muthén, 2019). The level-2 latent predictor $\beta_{0 i x}$ has been called a random intercept factor (e.g., Curran et al., 2012), a latent mean (e.g., Preacher et al., 2016) and a latent covariate (e.g., Lüdtke et al., 2008). The level-1 $x w_{\text {tix }}$ and $y w_{\text {tiy }}$ latent variables are also known as structured residuals (see Curran et al., $2012,2014)$, which are used to ensure that the $\gamma_{01}$ level-2 fixed effect of $\beta_{0 i}$ is a between-level effect as intended, and not a contextual effect (found as the BP intercept effect minus the WP residual effect; see Hoffman, 2019). In SEMs, $w_{t i x}$ and $y w_{t i y}$ are created as new latent variables (one per occasion) that absorb the observed variables' residuals and any fixed effects among them. Because $x w_{t i x}$ is unpredicted in Equation 4, its total variance is the same as its level-1 $e_{t i x}$ residual variance $\left(\sigma_{e_{x}}^{2}\right)$. In contrast, because part of the variance in $y w_{t i y}$ has been explained by a fixed effect of $x w_{t i x}$, its unexplained variance is the level-1 $e_{t i y}$ residual variance $\left(\sigma_{e_{y}}^{2}\right)$. 


\section{Cross-Lagged WP Residual Associations}

The conditional models thus far have all posited a unidirectional concurrent or lagged WP association—of $x_{t i}$ predicting $y_{t i}$. But also frequently of interest are cross-lagged effects, which are bidirectional lagged effects included to ascertain which variable is driving subsequent changes in the other. A model often used to examine such associations, the auto-regressive cross-lagged panel model (CPLM), is shown in single-level notation in Equation 5 below:

$$
\begin{aligned}
& x_{t i x}=\gamma_{t 0 x}+\gamma_{10 x}\left(x_{t-1 i}\right)+\gamma_{20 x}\left(y_{t-1 i}\right)+e_{t i x} \\
& y_{t i y}=\gamma_{t 0 y}+\gamma_{10 y}\left(y_{t-1 i}\right)+\gamma_{20 y}\left(x_{t-1 i}\right)+e_{t i y} \\
& \boldsymbol{R}:\left[\begin{array}{l}
e_{0 i x} \\
e_{1 i x} \\
e_{2 i x} \\
e_{3 i} \\
e_{4 i x} \\
e_{0 i y} \\
e_{1 i} \\
e_{2 i y} \\
e_{3 i y} \\
e_{4 i y}
\end{array}\right] \sim \mathrm{MVN}\left[\begin{array}{l}
0 \\
0 \\
0 \\
0 \\
0 \\
0 \\
0 \\
0 \\
0 \\
0
\end{array}\right],\left[\begin{array}{cccccccccc}
\sigma_{e_{x 0}}^{2} \\
0 & \sigma_{e_{x}}^{2} & 0 & 0 & 0 & 0 & \sigma_{x, y} & 0 & 0 & 0 \\
0 & 0 & \sigma_{e_{x}}^{2} & 0 & 0 & 0 & 0 & \sigma_{x, y} & 0 & 0 \\
0 & 0 & 0 & \sigma_{e_{x}}^{2} & 0 & 0 & 0 & 0 & \sigma_{x, y} & 0 \\
0 & 0 & 0 & 0 & \sigma_{e_{x}}^{2} & 0 & 0 & 0 & 0 & \sigma_{x, y} \\
\sigma_{x 0, y 0} & 0 & 0 & 0 & 0 & \sigma_{e_{y 0}}^{2} & 0 & 0 & 0 & 0 \\
0 & \sigma_{x, y} & 0 & 0 & 0 & 0 & \sigma_{e_{y}}^{2} & 0 & 0 & 0 \\
0 & 0 & \sigma_{x, y} & 0 & 0 & 0 & 0 & \sigma_{e_{y}}^{2} & 0 & 0 \\
0 & 0 & 0 & \sigma_{x, y} & 0 & 0 & 0 & 0 & \sigma_{e_{y}}^{2} & 0 \\
0 & 0 & 0 & 0 & \sigma_{x, y} & 0 & 0 & 0 & 0 & \sigma_{e_{y}}^{2}
\end{array}\right]
\end{aligned}
$$

in which the matrix $\boldsymbol{R}$ includes covariances for the cross-variable residuals at the same occasion, given that these concurrent relations are no longer included as fixed effects. The first occasion's residual variances and covariances are allowed to differ from those of subsequent occasions. As also shown in the corresponding SEM diagram in Figure 4a, the CPLM differs from the prior models by including fixed effects of the prior occasion $(t-1)$ that predict the current occasion, both within variables ( $\gamma_{10 x}$ and $\gamma_{10 y}$, the auto-regressive effects), as well as across variables ( $\gamma_{20 x}$ and $\gamma_{20}$, the cross-lagged effects). By convention both variables' fixed intercepts (timespecific means) are allowed to differ by occasion (as shown by the $t$ in the fixed intercepts $\gamma_{t 0 x}$ and $\gamma_{t 0 y}$ ), as could the other fixed effects, residual variances, and residual covariances. 


\section{— Insert Figure 4 about here -}

Unfortunately, this CPLM variant has the same problem of smushed effects of timevarying predictors as in Equation 2: it does not distinguish the BP intercept effects from WP residual effects of each variable in predicting the other. This is because the auto-regressive effects - included to control for stability over time-do so by predicting an exponential decay over time in the correlation across occasions, which will not be sufficient when individual mean differences are prominent (that create an additional pattern of time-constant correlation between occasions). To solve this problem, we can add a random intercept (RI) for each variable to create the RI-CPLM (Hamaker et al., 2015), as shown with single-level notation in Equation 6 below:

$$
\begin{aligned}
& x_{t i x}=\gamma_{t 0 x}+\gamma_{10 x}\left(x_{t-1 i}\right)+\gamma_{20 x}\left(y_{t-1 i}\right)+U_{0 i x}+e_{t i x} \\
& y_{t i y}=\gamma_{t 0 y}+\gamma_{10 y}\left(y_{t-1 i}\right)+\gamma_{20 y}\left(x_{t-1 i}\right)+U_{0 i y}+e_{t i y} \\
& \boldsymbol{G}:\left[\begin{array}{l}
U_{0 i x} \\
U_{0 i y}
\end{array}\right] \sim \operatorname{MVN}\left[\begin{array}{l}
0 \\
0
\end{array}\right],\left[\begin{array}{cc}
\tau_{U_{0 x}}^{2} & \tau_{U_{0_{x}}, U_{0 y}} \\
\tau_{U_{0_{x}}, U_{0 y}} & \tau_{U_{0 y}}^{2}
\end{array}\right]
\end{aligned}
$$

which only differs from the CLPM in Equation 5 by the new level-2 random intercepts $\left(U_{0 i x}\right.$ and $\left.U_{0 i y}\right)$, whose variances and covariance are shown in the new matrix $\boldsymbol{G}$. By convention, a covariance between random intercepts is estimated instead of a fixed effect - no directionality is specified. A corresponding SEM diagram is shown in Figure 4b. When person mean differences are the only source of BP variance in each variable, the RI-CPLM's cross-lagged effects will then reflect unconfounded WP residual associations as intended (see Berry \& Willoughby, 2017; Hamaker et al., 2015). RI-CPLMs can also be extended to include occasion-specific unique factors (for a full taxonomy of models for cross-lagged relations, see Usami et al., 2019).

\section{The Role of Individual Change over Time in Disaggregating BP Associations}

Thus far we have considered three different strategies for representing individual mean differences in a time-varying predictor. Two can be implemented in univariate longitudinal models by using its person mean or its baseline occasion as an observed level-2 predictor, as in 
Equation 3. The third requires multivariate longitudinal models by which to estimate its intercept to become a latent level-2 predictor, as in Equations 4 or 6 . So when would one expect these three ways of representing relations of BP mean differences to lead to different answers?

Let us first consider the scenario in which the only source of individual-level variance in a time-varying predictor is person mean differences (i.e., BP intercept variance). If so, then representing that BP mean variance using all occasions - as when using an observed or latent person mean - should work better than using only the baseline occasion, given that the latter contains relatively more WP residual variance (for a demonstration, see Hoffman, 2015, ch. 9). Further, using a latent person mean should work better than using an observed person mean, given that the latter is usually not perfectly reliable. Indeed, the level-2 fixed effects (BP intercept effects) of an observed person mean have downward bias relative to those for a latent person mean (e.g., Lüdtke et al., 2008; Preacher et al, 2011), particularly given fewer occasions and smaller ICCs for the predictor. Fortunately, level-1 fixed effects (WP residual effects) can be recovered equivalently well for an observed person mean deviation as for a latent residual.

But what if other sources of individual differences are present in a time-varying predictor, such as individually-varying change over time? The models presented thus far have ignored this possibility - in our example, while most models included an individual time slope for the $y_{t i}$ memory outcome, none included an individual time slope for the $x_{t i}$ speed predictor (or relations thereof). The impact of this omission depends on whether the effect of time in predicting speed is fixed or random. If it fixed only - there is change over time in $x_{t i}$ on average, but no individual differences in change - including the same fixed time trend in predicting memory will eliminate any bias, given that any time-varying predictor's level-1 fixed effect is always a unique effect controlling for covariance with other predictors (including time; see Wang \& Maxwell, 2015). 
Otherwise, if people differ randomly in their extent of change over time in a time-varying predictor, this creates two distinct problems. The first problem relates to its BP intercept effects, which must be interpreted conditionally given that individual change over time creates a distinct placement of persons at any cross-section in time. Consequently, results may differ across models that represent a predictor's BP intercept differences at different points in time (e.g., as when using the person mean versus using the baseline value of a time-varying predictor).

The second problem is more insidious - the level-1 predictor (created by centering the time-varying predictor with an observed mean, its observed baseline value, or a latent person mean) is supposed to reflect only WP residual variability, but it will also contain BP variability in change over time. That is, the level-1 predictor is really still two variables, and each can have different relations with a time-varying outcome. All of the models presented thus far would force both variables to share the same fixed effect, creating a second type of conflated level-1 effecta time-smushed effect, derivations for which using Equation 3 are provided in Appendix B. As shown in Equations B4 and B7, a time-smushed effect is created when the level-1 fixed effect is applied to not only the predictor's WP residual as intended, but also its BP individual time slope.

A time-smushed effect is a different misspecification than already addressed by adding a level-2 variable (observed person mean, observed baseline value, or latent person mean) for the time-varying predictor, which unconfounded its WP residual and BP intercept relations. Instead, a time-smushed effect conflates a WP residual relation with a BP time slope relation - the novel latter possibility is the extent to which individual time slopes in a time-varying predictor relate to those of a time-varying outcome. Here, a BP time slope association indicates the extent to which older adults who decline less in speed over time (relative to other persons) also decline less in memory over time. A purely WP residual association then represents the extent to which greater 
speed than predicted (now after controlling for individual change in speed over time) relates to higher memory than predicted (after controlling for individual change in memory over time), either at the same occasion (a concurrent relation) or at a subsequent occasion (a lagged relation).

\section{Univariate Longitudinal Models with Time-Detrended Level-1 Predictors}

One possible strategy for preventing time-smushed effects relies on an older, two-stage method known as slopes as outcomes, as described by Curran and Bauer (2011). They created time-detrended versions of time-varying predictors by estimating a separate regression model per person to predict the time-varying predictor from mean-centered time. The per-person intercepts and per-occasion residuals were then saved for use as observed $x_{t i}$ predictors of $y_{t i}$, such as in Equation 3. The level-1 time-detrended residual predictor should then capture only the WP effect, and the level-2 intercept predictor should capture only the BP intercept effect (of predictor differences at the mean occasion). Given balanced time, Curran and Bauer found that the bias in WP residual effects created by using PMC in univariate longitudinal models was mitigated using time-detrended level-1 predictors (and BP effects were recovered well either way given the use of mean-centered time to create per-person intercepts). As expected, these results did not hold as well given unbalanced time, in which differences in the occasions observed translated into differences in the time-varying $x_{t i}$ values observed (i.e., reflecting different means for time).

Time-detrended predictors in univariate longitudinal models were also addressed by Wang and Maxwell (2015). As noted earlier, they found that when using PMC, including a fixed time effect predicting a time-varying outcome sufficiently controlled for a fixed time effect in a time-varying predictor. They also concluded that a random time slope predicting a time-varying outcome sufficiently controlled for a random time slope in a time-varying predictor, and thus that time-detrending the level-1 predictor (when using PMC) was unnecessary. However, their 
simulation could not address potential confounding of WP residual and BP time slope relations, given that the individual time slope variance in their time-varying predictor was only $10 \%$ of that specified in their time-varying outcome. More importantly, no BP relation for the individual time slopes across variables was specified or examined (as was also true of Curran \& Bauer, 2011, whose simulated outcome did not have random time slope variance). The present study will expand on this previous work by manipulating conditions by which to directly demonstrate how WP residual and BP time slope relations will be confounded in each model presented thus far. — Insert Figure 5 about here -

\section{Univariate Longitudinal Models that Allow BP Associations in Time Slopes}

The use of time-detrending to remove individual time slope variance from a time-varying predictor is predicated on the idea that such time slope variance is an unwanted nuisance. But in many contexts, such time slopes and their BP associations are likely to be of direct theoretical interest. In such cases, rather than discarding the time slope coefficients created by the perperson regression models, one could conceivably include them as a third model predictor, as shown by expanding the univariate longitudinal model from Equation 3 into Equation 7 below:

$$
\begin{aligned}
& \text { WP Level-1 Model: } \quad \text { BP Level-2 Model: } \\
& y_{t i}=\beta_{0 i}+\beta_{1 i}\left(\text { Time }_{t i}\right) \quad \text { Intercept: } \quad \beta_{0 i}=\gamma_{00}+\gamma_{01}\left(x_{i}^{*}\right)+\gamma_{02}\left(x t_{i}^{*}\right)+U_{0 i} \\
& +\beta_{2 i}\left(x_{t i}^{*}\right)+e_{t i} \quad \text { Time Slope: } \quad \beta_{1 i}=\gamma_{10}+\gamma_{11}\left(x_{i}^{*}\right)+\gamma_{12}\left(x t_{i}^{*}\right)+U_{1 i} \\
& \text { WP } x_{t i}^{*} \text { Slope: } \beta_{2 i}=\gamma_{20}
\end{aligned}
$$

whose three predictors would be created from per-person regression models predicting $x_{t i}$ with baseline-centered Time $e_{t i}$ : level-1 $x_{t i}^{*}$ as the time-detrended residuals, level-2 $x_{i}^{*}$ as the perperson intercept, and level-2 $x t_{i}^{*}$ as the per-person time slope. A corresponding SEM diagram is shown in Figure 5a. At level 2, the four fixed effects allow initial differences and individual change over time in speed to each predict initial differences and change over time in memory.

\section{Multivariate Longitudinal Models that Allow BP Associations in Time Slopes}


Multivariate longitudinal models provide a simultaneous strategy for capturing individual change over time in time-varying predictors and outcomes. We can expand Equation 4-which already specified a latent intercept for the time-varying predictor-by adding its latent time slope and level-2 fixed effects predicting the time-varying outcome, as shown in Equation 8 below:

WP Level-1 Model:

$$
\begin{aligned}
x_{t i x} & =\beta_{0 i x}+\beta_{1 i x}\left(\text { Time }_{t i}\right)+x w_{t i x} \\
x w_{t i x} & =e_{t i x} \\
y_{t i y} & =\beta_{0 i y}+\beta_{1 i y}\left(\text { Time }_{t i}\right)+y w_{t i y} \\
y w_{t i y} & =\beta_{2 i y}\left(x w_{t i x}\right)+e_{t i y}
\end{aligned}
$$

BP Level-2 Model and Level-2 Matrix $\boldsymbol{G}$ :

$$
\begin{aligned}
& x_{\text {tix }} \text { Intercept: } \quad \beta_{0 i x}=\gamma_{00 x}+U_{0 i} \\
& x_{\text {tix }} \text { Time Slope: } \quad \beta_{1 i x}=\gamma_{10 x}+U_{1 i} \\
& y_{\text {tiy }} \text { Intercept: } \quad \beta_{0 i}=\gamma_{00 y}+\gamma_{01 y}\left(\beta_{0 i x}\right)+\gamma_{02 y}\left(\beta_{1 i}\right)+U_{0 i} \\
& y_{\text {tiy }} \text { Time Slope: } \quad \beta_{1 i y}=\gamma_{10 y}+\gamma_{11 y}\left(\beta_{0 i}\right)+\gamma_{12 y}\left(\beta_{1 i}\right)+U_{1 i y} \\
& y w_{\text {tiy }} \text { WP } x w_{\text {tix }} \text { Slope: } \beta_{2 i y}=\gamma_{20 y} \\
& \boldsymbol{G}:\left[\begin{array}{l}
U_{0 i x} \\
U_{1 i} \\
U_{0 i} \\
U_{1 i y}
\end{array}\right] \sim \operatorname{MVN}\left[\begin{array}{l}
0 \\
0 \\
0 \\
0
\end{array}\right],\left[\begin{array}{cccc}
\tau_{U_{0 x}}^{2} & \tau_{U_{0 x}, U_{1 x}} & - & - \\
\tau_{U_{0_{x}}, U_{1 x}} & \tau_{U_{1 x}}^{2} & - & - \\
- & - & \tau_{U_{0 y}}^{2} & \tau_{U_{0 y}, U_{1 y}} \\
- & - & \tau_{U_{0 y}, U_{1 y}} & \tau_{U_{1 y}}^{2}
\end{array}\right]
\end{aligned}
$$

in which the new individual time slope $\beta_{1 i x}$ (for change over time in the speed predictor) now has fixed effects $\gamma_{02 y}$ and $\gamma_{12 y}$ to predict the $\beta_{0 i}$ intercept and $\beta_{1 i y}$ time slope for memory. In the level-2 matrix $\boldsymbol{G}$, dashes again indicate the covariances captured by fixed effects instead; the level-1 matrix $\boldsymbol{R}$ remains as was given in Equation 4. A corresponding SEM diagram, shown in Figure $5 \mathrm{~b}$, depicts a new latent oval for the $\beta_{1 i x}$ individual time slope (and its two fixed effects).

Likewise, we can examine cross-lagged WP residual relations while also estimating individual time slopes for each variable. The RI-CPLM from Equation 6 - that previously had only random intercepts for each variable — can be expanded to include fixed and random time slopes for both variables, known as a latent curve model with structured residuals (LCM-SR; Curran et al. 2014), as shown in Equation 9 below: 
WP Level-1 Model:

$$
\begin{aligned}
x_{t i x} & =\beta_{0 i x}+\beta_{1 i x}\left(\text { Time }_{t i}\right)+x w_{t i x} \\
x w_{t i x} & =\beta_{2 i x}\left(x_{t-1 i}\right)+\beta_{3 i x}\left(y_{t-1 i}\right)+e_{t i x} \\
y_{t i y} & =\beta_{0 i}+\beta_{1 i y}\left(\text { Time }_{t i}\right)+y w_{t i y} \\
y w_{t i y} & =\beta_{2 i y}\left(y_{t-1 i}\right)+\beta_{3 i y}\left(x_{t-1 i}\right)+e_{t i y}
\end{aligned}
$$

BP Level-2 Model and Level-2 Matrix $\boldsymbol{G}$ :

$x_{\text {tix }}$ Intercept: $\quad \beta_{0 i x}=\gamma_{00 x}+U_{0 i x}$

$x_{\text {tix }}$ Time Slope: $\beta_{1 i x}=\gamma_{10 x}+U_{1 i}$

$y_{\text {tiy }}$ Intercept: $\quad \beta_{0 i y}=\gamma_{00 y}+U_{0 i y}$

$y_{\text {tiy }}$ Time Slope: $\beta_{1 i y}=\gamma_{10 y}+U_{1 i y}$

$x w_{t i x}$ WP AR $x w_{t i x}$ Slope: $\beta_{2 i x}=\gamma_{20 x}$

$x w_{t i x}$ WP CL $y w_{t i y}$ Slope: $\beta_{3 i}=\gamma_{30 x}$

$y w_{\text {tiy }}$ WP AR $y w_{\text {tiy }}$ Slope: $\beta_{2 i y}=\gamma_{20 y}$

$y w_{t i y}$ WP CL $x w_{t i x}$ Slope: $\beta_{3 i}=\gamma_{30 y}$

$\boldsymbol{G}:\left[\begin{array}{l}U_{0 i} \\ U_{1 i} \\ U_{0 i} \\ U_{1 i y}\end{array}\right] \sim \operatorname{MVN}\left[\begin{array}{l}0 \\ 0 \\ 0 \\ 0\end{array}\right],\left[\begin{array}{cccc}\tau_{U_{0 x}}^{2} & \tau_{U_{0_{x}}, U_{1 x}} & \tau_{U_{0_{x}}, U_{0 y}} & \tau_{U_{0_{x}}, U_{1 y}} \\ \tau_{U_{0_{x}}, U_{1 x}} & \tau_{U_{1 x}}^{2} & \tau_{\tau_{U_{1}, U_{0 y}}} & \tau_{U_{1_{x}}, U_{1 y}} \\ \tau_{U_{0_{x}}, U_{0}} & \tau_{U_{1_{x}}, U_{0 y}} & \tau_{U_{0 y}}^{2} & \tau_{U_{0_{y}}, U_{1 y}} \\ \tau_{U_{0_{x}}, U_{1 y}} & \tau_{U_{1_{x}}, U_{1 y}} & \tau_{U_{0_{y}}, U_{1 y}} & \tau_{U_{1 y}}^{2}\end{array}\right]$

in which two-level notation is used given the inclusion of random time slopes. The same level-1

matrix $\boldsymbol{R}$ is used as for the CLPM in Equation 5, but the level-2 matrix $\boldsymbol{G}$ has been expanded to include the variances and covariances of the random time slopes (with all covariances estimated given the omission of level-2 fixed effects for these relations). A corresponding SEM diagram is shown in Figure 4c. A fixed intercept and linear time slope for each variable have replaced the per-occasion intercepts $\gamma_{t 0 x}$ and $\gamma_{t 0 y}$ from Equations 5 and 6 , but nonlinear change could also be captured as needed (e.g., using estimated time slope factor loadings, as in a latent basis model).

The LCM-SR in Equation 9 has the benefit of directly capturing relations for individual changes over time, but there are alternative models that instead specify accumulating effects of the each variable's intercept and time slope, such as the auto-regressive latent trajectory model (e.g., Bollen \& Curran, 2004) and the latent change (or difference) score model (e.g., McArdle \& Hamagami, 2001). Although there are conditions under which these latter models are equivalent 
to the LCM-SR model (see Usami et al., 2019; Scott, 2020), models with accumulating effects are not pursued here, given the present goal of clearly differentiating the BP and WP levels in describing how variables relate over time. Likewise, not considered is the general cross-lagged panel model (Zyphur et al., 2020a, 2020b), in which factor loadings are estimated for the latent intercept (making it a more general person factor), but in which separate individual time slope factors are not included (and thus only one source of person dependency is assumed). Other ways of using general factors to replace time slope factors are possible as well (see Tuliao et al., 2017).

\section{Demonstrating Time-Smushed Effects via Simulation}

The impact of ignoring individual time slopes in a time-varying predictor and their BP associations is now illustrated using a simulation study. Given the amount of previous work on intercept-smushed effects (e.g., Berry \& Willoughby, 2017; Curran \& Bauer, 2011; Curran et al., 2012; Hamaker et al., 2015), models with this additional problem (Equations 2 and 5) were not included. The present focus on identifying time-smushed effects expands on previous research (Curran \& Bauer, 2011; Hoffman, 2015, ch. 9; Scott, 2020; Wang \& Maxwell, 2015) in three respects. First, it addresses both univariate longitudinal models (that include observed predictor variables created by PMC, BLC, or time-detrending) and multivariate longitudinal models (that include latent predictor variables). Second, it uses a design that can distinguish the impact of individual time slopes simply being present in a time-varying predictor from further bias caused by their unmodeled relationship with the individual time slopes of a time-varying outcome. Third, it evaluates the recovery of both concurrent and cross-lagged WP residual effects.

\section{Simulation Method and Results}

\section{Data Generation}

This simulation examined to what extent the previous models can recover three distinct 
relations across longitudinal variables: among their time-specific residuals (WP residual relation), among their individual intercepts (BP intercept relation), and among their individual time slopes (BP time slope relation). SAS was used to simulate $x_{t i x}$ and $y_{t i x}$ variables with no missing data for 100 persons at five occasions. These sample sizes were chosen to be small enough to reflect real-world longitudinal data, but large enough to prevent the downward bias of random effects variances arising from the use of maximum likelihood (ML) estimation (McNeish \& Stapleton, 2016). ML was chosen to maintain equivalence given that residual maximum likelihood (REML) is not available in SEM software. The univariate longitudinal models (Equations 3 and 7) were estimated using SAS MIXED. The multivariate longitudinal models (Equations 4, 8, and 9) were estimated using Mplus 8.4 (Muthén and Muthén, 1998-2017).

The population data were generated using a multivariate longitudinal model predicting both $x_{\text {tix }}$ and $y_{\text {tiy }}$ simultaneously, as shown in Equation 10 below:

WP Level-1 Model and Level-1 Matrix $\boldsymbol{R}$ :

$x_{t i x}=\beta_{0 i x}+\beta_{1 i x}\left(\right.$ Time $\left._{t i}\right)+e_{t i x}$
$y_{t i y}=\beta_{0 i y}+\beta_{1 i y}\left(\right.$ Time $\left._{t i}\right)+e_{t i y}$

$y_{t i y}=\beta_{0 i y}+\beta_{1 i y}\left(T i m e_{t i}\right)+e_{t i y}$

$\boldsymbol{R}:\left[\begin{array}{l}e_{0 i} \\ e_{1 i x} \\ e_{2 i x} \\ e_{3 i x} \\ e_{4 i x} \\ e_{0 i y} \\ e_{1 i y} \\ e_{2 i y} \\ e_{3 i y} \\ e_{4 i y}\end{array}\right] \sim \mathrm{MVN}\left[\begin{array}{l}0 \\ 0 \\ 0 \\ 0 \\ 0 \\ 0 \\ 0 \\ 0 \\ 0 \\ 0\end{array}\right],\left[\begin{array}{cccccccccc}\sigma_{e_{x}}^{2} & 0 & 0 & 0 & 0 & \sigma_{x, y} & 0 & 0 & 0 & 0 \\ 0 & \sigma_{e_{x}}^{2} & 0 & 0 & 0 & 0 & \sigma_{x, y} & 0 & 0 & 0 \\ 0 & 0 & \sigma_{e_{x}}^{2} & 0 & 0 & 0 & 0 & \sigma_{x, y} & 0 & 0 \\ 0 & 0 & 0 & \sigma_{e_{x}}^{2} & 0 & 0 & 0 & 0 & \sigma_{x, y} & 0 \\ 0 & 0 & 0 & 0 & \sigma_{e_{x}}^{2} & 0 & 0 & 0 & 0 & \sigma_{x, y} \\ \sigma_{x, y} & 0 & 0 & 0 & 0 & \sigma_{e_{y}}^{2} & 0 & 0 & 0 & 0 \\ 0 & \sigma_{x, y} & 0 & 0 & 0 & 0 & \sigma_{e_{y}}^{2} & 0 & 0 & 0 \\ 0 & 0 & \sigma_{x, y} & 0 & 0 & 0 & 0 & \sigma_{e_{y}}^{2} & 0 & 0 \\ 0 & 0 & 0 & \sigma_{x, y} & 0 & 0 & 0 & 0 & \sigma_{e_{y}}^{2} & 0 \\ 0 & 0 & 0 & 0 & \sigma_{x, y} & 0 & 0 & 0 & 0 & \sigma_{e_{y}}^{2}\end{array}\right]$

BP Level-2 Model and Level-2 Matrix $\boldsymbol{G}$ :

$x_{\text {tix }}$ Intercept: $\quad \beta_{0 i x}=\gamma_{00 x}+U_{0 i x}$

$x_{\text {tix }}$ Time Slope: $\beta_{1 i x}=\gamma_{10}+U_{1 i x}$

$y_{\text {tiy }}$ Intercept: $\quad \beta_{0 i y}=\gamma_{00 y}+U_{0 i y}$

$y_{\text {tiy }}$ Time Slope: $\beta_{1 i y}=\gamma_{10 y}+U_{1 i y}$ 


$$
\boldsymbol{G}:\left[\begin{array}{l}
U_{0 i x} \\
U_{1 i x} \\
U_{0 i y} \\
U_{1 i y}
\end{array}\right] \sim \operatorname{MVN}\left[\begin{array}{l}
0 \\
0 \\
0 \\
0
\end{array}\right],\left[\begin{array}{cccc}
\tau_{U_{0 x}}^{2} & 0 & \tau_{U_{0_{x}}, U_{0 y}} & 0 \\
0 & \tau_{U_{1 x}}^{2} & \tau_{\tau_{U_{1 x}, U_{0}}} & \tau_{U_{1_{x}}, U_{1 y}} \\
& 0 & \tau_{U_{0 y}}^{2} & 0 \\
\tau_{U_{0_{x}}, U_{0 y}} & 0 & 0 & \tau_{U_{1 y}}^{2} \\
0 & \tau_{U_{1_{x}}, U_{1 y}} & 0
\end{array}\right]
$$

in which all terms are defined similarly as in the prior models. For ease of interpretation $x_{\text {tix }}$ and $y_{\text {tiy }}$ had a common scale $\left(M=0, S D=1, \mathrm{ICC}=.60\right.$ at Time $\left._{t i}=0\right)$. This ICC reflects real-world longitudinal data, in which BP mean differences tend to be large. Also held constant were the fixed intercepts $\left(\gamma_{00 x}, \gamma_{00 y}=0\right)$, the level-1 residual variances $\left(\sigma_{e_{x}}^{2}, \sigma_{e_{y}}^{2}=0.40\right)$, the level-2 random intercept variances $\left(\tau_{U_{0 x}}^{2}, \tau_{U_{0 y}}^{2}=0.60\right)$, and the level-2 random time slope variances $\left(\tau_{U_{1 x}}^{2}\right.$, $\left.\tau_{U_{1 y}}^{2}=.06\right)$. The latter were created by specifying 0.60 for time slope reliability, the proportion of variance attributable to individual time slope differences relative the total longitudinal variance (analogous to true score over total variance; see Willett, 1989), as shown in Equation 11 below:

$$
\text { Slope Variance }=\frac{\text { Slope Reliability } * \frac{\sigma_{e}^{2}}{S S T}}{1-\text { Slope Reliability }}=\frac{0.6 * \frac{0.4}{10}}{1=0.6}=0.06
$$

in which $S S T=10$ is the sum of squares for time for the five equidistant occasions. A slope reliability of 0.60 was chosen to create random time slope variances that were large enough to avoid convergence problems but small enough to be realistic for only five occasions.

\section{Manipulated Factors}

The study design manipulated five factors yielding 32 total conditions; 500 replications were generated for each condition. Three factors reflected across-variable correlations: of the level-1 concurrent residuals, the level-2 random intercepts, and the level-2 random time slopes. Each correlation was specified as 0 or .3 (then translated into the covariances used in Equation 10). These three factors were included to demonstrate the presence of time-smushed fixed effects of $x_{t i x}$ in predicting $y_{t i y}$. As for the other two factors, the two fixed time slopes $\left(\gamma_{10 x}\right.$ and $\left.\gamma_{10 y}\right)$ 
were specified as 0 or 0.16 - these were included solely to demonstrate their lack of impact.

Although the three relations of $x_{t i x}$ with $y_{t i y}$ were generated as covariances in Equation 10 , some of the analysis models will address the scenario of $x_{t i x}$ as a predictor of $y_{t i y}$, in which those covariances are represented using directed fixed effects instead. For example, in expressing the covariances in Equation 10 using directed fixed effects in Equation 8, the WP level-1 residual covariance $\left(\sigma_{x, y}\right)$ becomes $\gamma_{20 y}$ for the $x_{t i x}$ level- 1 residual predicting the $y_{t i y}$ level-1 residual, the BP level-2 intercept covariance $\left(\tau_{U_{0 x}, U_{0 y}}\right)$ becomes $\gamma_{01 y}$ for the $x_{t i x}$ level-2 intercept predicting the $y_{\text {tiy }}$ level-2 intercept, and the BP level-2 time slope covariance $\left(\tau_{U_{1_{x}}, U_{1 y}}\right)$ becomes $\gamma_{11 y}$ for the $x_{t i x}$ level-2 time slope predicting the $y_{t i y}$ level-2 time slope. Because $x_{t i x}$ and $y_{t i y}$ share a common scale and variance components, signed raw bias in each fixed effect (relative to its corresponding population correlation) indicated misspecification in models in which all three $x_{\text {tix }}$ predictor variables were included (level-1 residual, level-2 intercept, and level-2 time slope). Wald tests were used to examine Type I error for the fixed effects that corresponded to a true correlation $=0$. For models without a level-2 time slope predictor (Equations 3 and 4), the impact of its missing time slope relation $\left(\gamma_{11 y}\right.$ for $\left.\tau_{U_{1 x}, U_{1 y}}\right)$ was indexed by pseudo- $R^{2}$ for the level-2 time slope variance in $y_{\text {tiy }}$ that it should have explained (i.e., its proportion reduction relative to the unconditional model for change over time in Equation 1; Singer \& Willett, 2003, ch. 4).

To examine the main effects and interactions of all five factors, bias was predicted at the replication level using analysis of variance to generate semi-partial $\eta^{2}$ effect sizes. As expected, the presence or absence of the two fixed time slopes made no difference-their combined main effects and interactions accounted for a maximum of $0.22 \%$ of the variance in obtained bias from any analysis model. This is because each model included a fixed time slope for $y_{\text {tiy }}$, by which 
each level-1 WP effect of $x_{t i x}$ became a unique effect controlled for any covariance with the Time $e_{t i}$ predictor (as described earlier; see also Wang \& Maxwell, 2015). After removing three replications that did not converge in all models, the replications were aggregated into 8 condition means (across the three types of relations) to examine bias within each model, as reported below. Simulation Results for Models with Concurrent WP Residual Associations

\section{Models Omitting Individual Time Slope Associations}

— Insert Figure 6 about here -

First examined were three models including directed BP intercept effects of $x_{\text {tix }}$ (using an observed person mean or baseline value in Equation 3, or a latent person mean in Equation 4), none of which included $x_{t i x}$ individual time slopes as a distinct variable in predicting the $y_{t i y}$ intercepts and time slopes. Patterns of bias in the three models are shown in Figure 6Figure 6, in which the columns distinguish the true BP correlation of the individual time slopes $\left(\mathrm{BP} r_{\text {time }}=0\right.$ or .3), while the x-axes distinguish the other true correlations: of the WP concurrent residuals $\left(\mathrm{WP} r_{\text {res }}=0\right.$ or .3$)$ and BP individual intercepts (BP $r_{\text {int }}=0$ or .3$)$. Separate rows depict each parameter; for clarity, $y$ subscripts are included in labeling each fixed effect predicting $y_{t i y}$.

The first row in Figure 6 shows bias in $\gamma_{01 y}$ (for level-2 $x_{i}^{*}$ or $\beta_{0 i x}$ predicting the $\beta_{0 i y}$ intercept) which should have captured only the intercept correlation (BP $\left.r_{\text {int }}\right)$. But in each model $\gamma_{01 y}$ also captured WP $r_{r e s}$, as seen by its positive bias when BP $r_{i n t}=0$ and WP $r_{r e s}=.3$, but negative bias when BP $r_{\text {int }}=.3$ and $\mathrm{WP} r_{\text {res }}=0$. As also shown in Appendix B, these results demonstrate that when $x_{t i x}$ contains individual time slope variance, its level-2 predictors that assume BP intercept variance only provide an incomplete separation of its BP intercept and WP residual effects (even if the $x_{t i x}$ individual time slopes are unrelated to those of $y_{t i y}$, as in the left column). For BP $r_{i n t}=0$, this bias created Type I error rates $\leq 20 \%$ for the observed person 
mean, $\leq 27 \%$ for the observed baseline, and $\leq 11 \%$ for the latent person mean. The second row shows bias in $\gamma_{11 y}$ (for level-2 $x_{i}^{*}$ or $\beta_{0 i x}$ predicting the $\beta_{1 i y}$ time slope), which should have always been 0 , as found using the observed baseline (at which point the $x_{\text {tix }}$ individual time slope has not yet contributed to its variability). In contrast, when using the observed or latent person mean, $\gamma_{11}$ was biased because it provided the "wrong" version of the $x_{t i x}$ intercept, given that person mean differences over time reflect not only BP intercept differences, but also BP time slope differences (as also shown in Appendix B). The latter manifested in the two conditions where BP $r_{\text {time }}$ differed from WP $r_{r e s}$ (with Type I error rates for $\gamma_{11 y} \leq 18 \%$ ).

To understand the direction of the $\gamma_{11 y}$ bias in these conditions, we turn to the third row in Figure 6, which presents bias in $\gamma_{20 y}$ (for level-1 $x_{t i}^{*}$ or $x w_{t i x}$ predicting the $y_{t i y}$ residual). Although $\gamma_{20 y}$ should have captured only the concurrent residual correlation (WP $r_{r e s}$ ), it captured the other relations as well. First, $\gamma_{20 y}$ was perturbed by BP $r_{\text {time }}$, as seen by its negative bias when WP $r_{r e s}=.3$ and $\mathrm{BP} r_{\text {time }}=0$, but positive bias when WP $r_{r e s}=0$ and $\mathrm{BP} r_{\text {time }}=$ .3. Bias was greater when using the observed baseline (Type I error rates $\leq 25 \%$ ) than when using the observed or latent mean (Type I error rates $\leq 13 \%$ ). Second, $\gamma_{20 y}$ was perturbed by BP $r_{\text {int }}$, as seen by its slight positive bias when BP $r_{\text {time }}=0$, WP $r_{\text {res }}=.0$, and BP $r_{\text {int }}=.3$, but negative bias when $\mathrm{BP} r_{\text {time }}=.3, \mathrm{WP} r_{\text {res }}=.3$, and $\mathrm{BP} r_{\text {int }}=0$. This result reflects the same incomplete separation of BP intercept from WP residual effects as was found for $\gamma_{01 y}$ (first row). Lastly, because none of these models included an $x_{t i x}$ time slope predictor to capture BP $r_{\text {time }}$, pseudo- $R_{U_{1 y}}^{2}$ for the $y_{t i y}$ time slope variance $\left(\tau_{U_{1 y}}^{2}\right)$ should be only slightly positive given the inclusion of level-2 $\gamma_{11 y}$. In the fourth row of Figure 6 , the expected pseudo- $R_{U_{1 y}}^{2} \approx .02$ is shown by the left-most condition in which all correlations $=0$. Nevertheless, $R_{U_{1 y}}^{2}$ shows that BP 
$r_{\text {time }}$ was indeed being captured, albeit inaccurately via time-smushed effects. Conflation of the BP $r_{\text {time }}$ effect with the WP $r_{r e s}$ effect (with both contributing to level-1 $\gamma_{20 y}$ ) is shown in the left column (BP $\left.r_{\text {time }}=.0\right)$ by a negative pseudo- $R_{U_{1 y}}^{2}$ when WP $r_{r e s}=.3$, as well as in the right column (BP $\left.r_{\text {time }}=.3\right)$ by a smaller pseudo- $R_{U_{1 y}}^{2}$ when WP $r_{r e s}=0$ than when WP $r_{r e s}=.3$

\section{Models Including Individual Time Slope Associations}

The results thus far demonstrate the need to distinguish BP time slope differences from BP intercept differences in time-varying predictors, even when the predictor's time slopes are unrelated to those of the outcome. We now consider the two ways to do so previously described: the two-stage univariate longitudinal model with time-detrended predictors (Equation 7) and the multivariate longitudinal model (Equation 8). For the two-stage variant, per-person single-level models regressing time-varying $x_{t i}$ on Time $_{t i}$ (estimated using ML in SAS MIXED) were first used to create the time-detrended predictors (which were then included in Equation 7).

\section{— Insert Figure 7 about here -}

Patterns of bias for the two models with three $x_{t i}$ predictors-level-1 residual, level-2 intercept, and level-2 time slope - are shown in Figure 7, in which the columns again distinguish the true BP $r_{\text {time }}$, while the x-axes distinguish the true WP $r_{r e s}$ and BP $r_{\text {int }}$. Separate rows depict each of the level-2 fixed effects. Notably, no bias was found in the level-1 WP effect in either model. Across rows, only minimal bias was exhibited by the multivariate longitudinal model (Equation 8; black lines); this is as expected given that it was equivalent to the population model.

The same was not true of the two-stage model, which exhibited the same problems found previously. In the first row, $\gamma_{01 y}$ (for the level- $2 x_{i}^{*}$ intercept predicting the $\beta_{0 i y}$ intercept) reflected not only BP $r_{\text {int }}$ as intended, but also WP $r_{\text {res }}$ (Type I error rates $\leq 11 \%$ ). In the second row, $\gamma_{11}$ (for $x_{i}^{*}$ predicting the $\beta_{1 i y}$ time slope) was biased when WP $r_{r e s} \neq \mathrm{BP} r_{\text {time }}$ (Type I 
error rates $\leq 9 \%$ ). In the third row, $\gamma_{02 y}$ (for the level-2 $x t_{i}^{*}$ time slope predicting the $\beta_{0 i y}$ intercept, which should have always been 0 ) had compensatory bias for $\gamma_{01 y}$ (Type I error rates $\leq$ $11 \%$ ). In the fourth row, $\gamma_{12 y}$ (for $x t_{i}^{*}$ predicting the $\beta_{1 i}$ time slope) captured not only BP $r_{\text {time }}$ as intended, but also WP $r_{\text {res }}$ (Type I error rates $\leq 18 \%$ ). In summary, unlike the latent $x_{\text {tix }}$ predictors in the simultaneous multivariate longitudinal model (Equation 8), the level-2 fixed effects of the observed $x_{\text {tix }}$ predictors in the two-stage univariate model (Equation 7) did not accurately distinguish the true BP relations of the $x_{t i x}$ intercept and time slope with those of $y_{t i}$.

\section{Simulation Results for Models with Cross-Lagged WP Residual Associations}

To also demonstrate the impact of omitting $x_{t i x}$ individual time slopes on recovery of cross-lagged WP effects, the model in Equation 9 was restricted in two ways. First was whether $x_{\text {tix }}$ was predicted by a $U_{1 i x}$ random time slope or by only a $U_{0 i x}$ random intercept; the model included all level-2 covariances then possible. Second was whether both sets of auto-regressive (AR) level-1 effects $\left(\gamma_{20 x}\right.$ and $\left.\gamma_{20 y}\right)$ were also included. All four models included a $U_{1 i y}$ random time slope for $y_{t i y}$, a $\gamma_{10 x}$ fixed time slope for $x_{t i x}$, and a concurrent residual covariance $\left(\sigma_{x, y}\right)$.

Results are shown in Figure 8, in which the columns again distinguish the true BP $r_{\text {time }}$, while the x-axes distinguish the true WP $r_{\text {res }}$ and BP $r_{i n t}$. Separate rows depict the four level-1 AR and cross-lag fixed effects, which were each 0 in the population. As expected, given its proper disaggregation of all BP sources of variance, no bias for any effect was found in the models predicting $x_{t i x}$ by a random time slope (in black lines). Otherwise, for models with only a random intercept for $x_{t i x}$ (in gray lines), the pattern of bias varied by the type of level-1 effect.

In the first row, the $y_{\text {tiy }}$ AR effect $\gamma_{20 y}$ had little bias; this was as expected given that the random time slope for $y_{t i y}$ was always included. But in the second row, omitting the random time slope for $x_{t i x}$ created large positive bias for the $x_{t i x}$ AR effect $\gamma_{20 x}$ across conditions 
(biases of $0.44-0.47$ were truncated for display at 0.20 ; Type I error rates $\leq 96 \%$ ). In the third row, the $x_{\text {tix }}$ to $y_{\text {tiy }}$ cross-lag effect $\gamma_{30 y}$ was biased by BP $r_{\text {time }}$ (more so when including the AR effects; Type I error rates $\leq 38 \%$ ). In the fourth row, bias for the $y_{\text {tiy }}$ to $x_{\text {tix }}$ cross-lag effect $\gamma_{30 x}$ differed by model. Without both AR effects (dashed lines), $\gamma_{30 x}$ was biased towards the missing BP $r_{\text {time }}$ and WP $r_{\text {res }}$ (Type I error rates $\leq 36 \%$ ). With both AR effects (solid lines), $\gamma_{30 x}$ was biased towards the missing BP $r_{\text {time }}$ when WP $r_{r e s} \neq \mathrm{BP} r_{\text {time }}$ (Type I error rates $\leq$ $25 \%$ ). In summary, adding AR effects did not prevent time-smushing of the cross-lagged level-1 effects: omitting the $x_{\text {tix }}$ individual time slopes created completely artificial cross-lagged effects.

\section{Results for Models using Motivating Example Data}

We now return to the motivating example: of processing speed predicting memory across five occasions in 470 older adults. Results from Robitaille et al. (2013) were adapted to simulate an example data set (using Equation 10) with which to illustrate the previous models. For convenience, no baseline age variance or attrition was included, and both variables had 0 fixed intercepts. Both variables declined on average over time, with significant individual differences in their intercepts and time slopes. These simulated data, along with syntax and complete output (in SAS and Mplus) for the models reported below, are provided in the online supplement.

— Insert Table 1 about here -

To begin, a single (smushed) level-1 effect of speed predicting memory (Equation 2) was estimated as $1.202(\mathrm{SE}=0.079)$. Results for the relations of speed with memory given by the other models are shown in Table 1. After adding fixed effects for the level-2 speed person mean (observed mean in Equation 3 PMC or latent mean in Equation 4), the level-1 fixed effect (as labeled speed residual predicting memory residual) was substantially reduced (0.571) — this is because it was no longer conflated with the level-2 effect of the speed intercept predicting the 
memory intercept. As expected, the latter was stronger when estimated using a latent mean (2.341) than a person mean (2.223). This BP intercept relation indicates that older adults with greater initial speed (than other adults) were predicted to have greater initial memory, as well as less decline in memory over time (as labeled speed intercept predicting memory time slope).

Equation 8 then unconfounds the time-smushed level-1 effect by including a latent time slope for speed, as well its fixed effects predicting memory. As in the true model, the speed time slope was significantly positively related to the memory time slope (4.419). This BP time slope relation indicates that adults with less decline in speed (than other adults) were predicted to have less decline in memory. The level-1 fixed effect of speed now accurately reflects a concurrent association of their WP residuals (0.411), as was also found using time-detrended residuals in Equation 7. However, the two-stage intercepts and slopes provided very different results for the level-2 fixed effects involving the time slopes (a discrepancy also found in the simulation).

Finally, in creating these data, auto-regressive or cross-lagged effects were not included (as not examined in Robitaille et al., 2013). In examining the results for the CLPMs-without random effects in Equation 5, adding random intercepts in Equation 6, and adding random time slopes in Equation 9- only the latter accurately recovered the true nonsignificant relations.

\section{Discussion}

The present article sought to expand on previous work addressing the conflation of predictor effects across levels of analysis in longitudinal data (e.g., Berry \& Willoughby, 2017; Curran \& Bauer, 2011; Curran et al., 2012, 2014; Hamaker et al., 2015; Hoffman, 2015, ch. 8-9, 2019; Scott, 2020; Wang \& Maxwell, 2015). Although the problems of conflated BP intercept and WP residual effects (as termed intercept-smushed effects here) are well-known, less attention has been paid to the conflation of BP time slope and WP residual effects (as termed 
time-smushed effects here). After explaining the reasons for this problem and its underpinnings (see Appendix B), a simulation study demonstrated that failing to account for individual time slopes in a time-varying predictor — as well as their relations with those of an outcome-can result in an incomplete disaggregation of their BP and WP associations. Notably, this problem manifested in models with concurrent or cross-lagged versions of WP residual relations.

Of the models considered here that included individual time slopes in a time-varying predictor, accurate results were obtained only from multivariate longitudinal models, in which the time-varying predictor's intercept and time slopes were each given latent variables, the same as the time-varying outcome. Although the two-stage univariate longitudinal model (with timedetrended predictors) did prevent time-smushed level-1 WP relations, the level-2 intercept and time slope relations were not recovered accurately, and thus a two-stage approach cannot be recommended. In contrast to previous advice that the decision whether or not to detrend time should be left up to the researcher (Wang \& Maxwell, 2015), an alternative view is suggested by the current study (Appendix B and simulation). Namely, if different results are found before distinguishing BP a time-varying predictor's time slope relations than after, this finding directly indicates model misspecification due to time-smushed effects (and thus uncertain accuracy of the BP intercept and WP residual effects found before isolating each variable's individual time slopes). The remainder of this paper provides recommendations for how to avoid time-smushed effects in practice and returns to the complications foreshadowed earlier for unbalanced time and random WP effects. These recommendations focus only on how to properly disaggregate sources of BP and WP associations among longitudinal variables; they do not address the additional conditions that would need to hold in order to infer causal relations of either variable in predicting the other (for a detailed treatment, see Usami et al., 2019; Zyphur et al., 2020a, 202b). 


\section{Recommendations for Practice}

To avoid smushed effects, the first step in any longitudinal analysis is to examine the sources of variance in every variable measured repeatedly over time, including those thought of as predictors. Which sources of BP variance are present is an empirical question to be resolved by modeling the predictor as an outcome; a more complete treatment of these steps is given in many sources (e.g., Hoffman, 2015, ch. 6; Singer \& Willett, 2003, ch. 4). Briefly, this process involves inspecting mean trajectories to decide what type of functional form may be most useful to describe change over time given the study context (i.e., continuous or discontinuous slopes, each linear or nonlinear). One would then test the fixed effects related to time needed to create the function, as well as the extent to which each effect shows individual differences (i.e., random effects). It's important to note that if the functional form chosen is not adequate to capture the observed change over time, the same problems of time-smushed effects could still result from incomplete description of individual differences in the effects of time. For example, if only a random linear model of change is fitted but random quadratic change is also present (i.e., people differ in their deceleration of linear change over time), those unmodeled individual quadratic time slopes could still result in an incomplete disaggregation of time-varying predictor effects.

It's important to note that these initial modeling steps to explore the impact of time are necessary regardless of the sampling time frame. That is, time-related effects may be present even in short-term studies over days or hours (Lui \& West, 2015); the present study highlights the need to consider their individual differences as well. Consider an example of daily measures for health and negative mood. Without a study treatment, an effect for time in study may not be useful. However, cycles for day of the week (e.g., for a worsening on Mondays) may still occur, individual differences in which can relate to those of other variables. If individual Monday 
effects in health correlate with those of mood, any examination of how mood predicts health (or vice-versa) must distinguish this relationship from the others possible: the BP intercept effect of being a grumpier person (which will differ on Mondays than other days), and the WP residual effect of being grumpier than usual (after taking into account if it's a Monday). Alternatively, if reactivity to the measurement process is evident, an exponential (or piecewise) time trend by survey exposure may capture differences in the initial occasions that dissipate over time. The context of the study and its measured constructs should help guide analysts to consider what aspects of time should be most relevant (e.g., time in study, hour of day, day of week), but the key idea is that the current findings logically apply to BP differences in any effect of time.

\section{Pros and Cons of Univariate and Multivariate Longitudinal Models}

If individually-varying time slopes are not found in an $x_{t i}$ time-varying predictor, then it will be sufficient to allow whatever fixed time effects are present in $x_{t i}$ to predict $y_{t i}$. The BP intercept and WP residual variables within $x_{t i}$ could then be parsed using observed predictors, as using PMC in the current study and in others (e.g., Curran \& Bauer, 2011; Wang \& Maxwell, 2015). Yet multivariate longitudinal models may still be preferred to univariate longitudinal models using PMC — even in the absence of $x_{t i}$ individual time slopes — for two reasons. First, as shown earlier, the level-2 effects of an observed person mean have a downward bias relative to the same effects estimated for a latent person mean, particularly given few occasions and small predictor ICCs (Lüdtke et al., 2008; Preacher et al, 2011). Second, predictors that are not included in the model likelihood (as is required in software for univariate longitudinal models, such as MIXED in SAS, SPSS, or STATA; nlme or lme4 in the R environment) cannot have missing data — cases that are incomplete are removed from an analysis, resulting in a loss of power at a minimum. The option to bring predictors into the likelihood within multivariate 
longitudinal models (using SEM software) prevents such case-wise deletion (albeit at the cost of invoking distributional assumptions for them). It also resolves ambiguities about how to form observed level-2 predictors with missing data, an issue side-stepped here by using complete data in the motivating example and in the simulation. For instance, given different patterns of missing time-varying predictors, should the person means be formed from all available occasions, or only occasions whose level-1 predictors will be included in the resulting model(s)? A difference between those strategies likely points to a missing data mechanism that should not be ignored (and thus which would benefit from the use of multivariate longitudinal models).

Multivariate longitudinal models with directed effects among outcomes (as time-varying predictors then become) usually require SEM software, which invokes additional estimation complexities. One is that, unlike multilevel software for univariate longitudinal models, SEM software does not have REML estimation (or denominator degrees of freedom in significance testing for fixed effects). In small samples of persons this limitation may result in downwardlybiased random effects variances coupled with greater rates of Type I errors in the corresponding fixed effects (McNeish \& Stapleton, 2016). A second complication is that testing moderation of intercept or time slope effects entails estimating interactions among latent variables, which requires numeric integration or other computationally-demanding methods. Thus, analysts may face practical limitations for the complexity of their longitudinal models with latent predictors in a multivariate framework relative to models with observed predictors in a univariate framework.

\section{Modeling Balanced versus Unbalanced Time}

This study's simulated data had two simplifying design constraints: the occasions were equidistant, and all persons shared the same possible occasions (i.e., balanced time). Fortunately, differing occasions across persons (unbalanced time) are readily accommodated in longitudinal 
models that use stacked data by altering the Time $_{t i}$ predictor to capture whatever interval has passed between occasions. In contrast, unbalanced time can have a larger impact in single-level SEMs that use a wide data structure (in which Time $_{t i}$ is not a single variable). Although in some software programs unbalanced time can be addressed using design variables for individuallyvarying factor loadings (e.g., TSCORES in Mplus), there is no analogous way to map the actual observed timing onto the separate time-varying predictor columns. Consequently, given severely unbalanced time across persons, it can be difficult in single-level SEMs to accurately specify many variants of WP effects. These include WP residual effects that vary across distinct time periods (i.e., that also don't map neatly onto the time-varying predictor columns; see McNeish \& Matta, 2020), auto-regressive and cross-lagged WP effects that refer to specific intervals, or random slopes across persons for the WP residual effect of other time-varying predictors besides time (and individual predictors thereof formed through cross-level interactions). The extent of bias created by treated unbalanced time as balanced will depend greatly on the context of the research. For instance, differences across persons in the timing of their observations by 1-2 months will likely not matter when measuring adults once a year, but such variation will almost certainly matter when measuring infant behavior (or other quickly developing phenomena).

Severely unbalanced time also makes it more challenging to diagnose absolute model misfit - a mismatch between the predicted occasion means, variances, and covariances to those of the data (i.e., from a saturated model). More specifically, the fixed intercept and time effects should recreate the occasion means, and the variances and covariances of the WP residuals and the BP random effects should recreate the occasion variances and covariances. Although relative gains in fit between models can always be examined, absolute fit is only testable when all persons share a common mean vector and covariance matrix - as for balanced time. This is why 
the absolute fit tests routinely provided for SEMs disappear when including individually-varying factor loadings or random slopes (that create person-specific covariance matrices). But because absolute fit tests do not capture effect sizes, they may not help in diagnosing intercept-smushed or time-smushed predictor effects (although see Scott, 2020, for the use of fit statistics in deciding amongst models for cross-lagged effects). In addition, although modification indices may suggest that residual variances should differ or residual covariances should be added, they will never recognize the need for a new latent variable for individual time slopes that may drive such residual modifications (i.e., that result from an incomplete model for person dependency).

\section{Conclusion}

In summary, the present work aimed to bring attention to the deleterious impacts of failing to represent BP variance in individual time slopes as a distinct part of a time-varying predictor. The resulting problem of time-smushed effects can occur in either univariate longitudinal models (i.e., using observed predictors as typically estimated using multilevel software) or multivariate longitudinal models (i.e., using latent predictors in single-level or multilevel SEM software). The present results suggest this disaggregation problem is best solved by longitudinal analysis within a multivariate framework by estimating latent variables for both time-varying predictors and time-varying outcomes (each of which can then predict each other directionally or merely covary, as desired by the researcher). The process of fitting one's study design to the options and associated terminology for doing so can be a confusing endeavor, in part because of the speed with which new model variants are continually being developed. I hope the present work has provided new motivation for an expanded treatment of the effects of time in time-varying predictors, as well as the practical direction by which to do so. 


\section{References}

Algina, J., \& Swaminathan, H. (2011). Centering in two-level nested designs. In J. Hox \& J. K. Roberts (Eds.), Handbook of advanced multilevel analysis (pp. 285-312). Taylor \& Francis.

Asparouhov, T. \& Muthén, B. O. (2019). Latent variable centering of predictors and mediators in multilevel and time-series models. Structural Equation Modeling, 26(1), 199-142. https://doi.org/10.1080/10705511.2018.1511375

Berry, D., \& Willoughby, M. (2017). On the practical interpretability of cross-lagged panel models: Rethinking a developmental workhorse. Child Development, 88(4), 1186-1206. https://doi.org/10.1111/cdev.12660

Bollen, K. A., \& Curran, P. J. (2004). Autoregressive latent trajectory (ALT) models: A synthesis of two traditions. Sociological Methods and Research, 32(3), 336-383. http://dx.doi.org/10.1177/0049124103260222

Curran, P. J., \& Bauer, D. J. (2011). The disaggregation of within-person and between-person effects in longitudinal models of change. Annual Review of Psychology, 62, 583-619. https://doi.org/10.1146/annurev.psych.093008.100356

Curran, P. J., Lee, T., Howard, A. L., Lane, S., \& MacCallum, R. A. (2012). Disaggregating within-person and between-person effects in multilevel and structural equation growth models. In J. R. Harring \& G. R. Hancock (Eds.), Advances in longitudinal methods in the social and behavioral sciences (pp. 217-253). Information Age.

Curran, P. J., Howard, A. L., Bainter, S. A., Lane, S. T., \& McGinley, J. S. (2014). The separation of between-person and within-person components of individual change over time: A latent curve model with structured residuals. Journal of Consulting and Clinical 
Psychology, 82(5), 879-894. https://doi.apa.org/doi/10.1037/a0035297

Hamaker, E. L., Kuiper, R. M., \& Grasman, R. P. P. P. (2015). A critique of the cross-lagged panel model. Psychological Methods, 20(1), 102-116. https://doi.org/10.1037/a0038889

Hoffman, L. (2012). Considering alternative metrics of time: Does anybody really know what "time" is? In J. Harring \& G. Hancock (Eds.), Advances in longitudinal methods in the social and behavioral sciences (pp. 255-287). Information Age.

Hoffman, L. (2015). Longitudinal analysis: Modeling within-person fluctuation and change. Routledge.

Hoffman, L. (2019). On the interpretation of multivariate multilevel model parameters across different combinations of model specification and estimation. Advances in Methods and Practices in Psychological Science,2(3), 288-311. https://psycnet.apa.org/doi/10.1177/2515245919842770

Hoffman, L., \& Stawski, R. S. (2009). Persons as contexts: Evaluating between-person and within-person effects in longitudinal analysis. Research in Human Development, 6(2-3), 97-210. https://doi.org/10.1080/15427600902911189

Liu, Y., \& West, S. G. (2015). Weekly cycles in daily report data: An overlooked issue. Journal of Personality, 84(5), 560-579. https://doi.org/10.1111/jopy.12182

Lüdtke, O., Marsh, H. W., Robitzsch, A., Trautwein, U., Asparouhov, T., \& Muthén, B. (2008). The multilevel latent covariate model: A new, more reliable approach to group-level effects in contextual studies. Psychological Methods, 13(3), 203-229. https://psycnet.apa.org/doi/10.1037/a0012869

McArdle, J. J., \& Hamagami, F. (2001). Latent difference score structural models for linear dynamic analyses with incomplete longitudinal data. In L. Collins \& A. Sayer (Eds.), 
New methods for the analysis of change (pp. 137-175). American Psychological Association.

McNeish, D. \& Matta, T. H. (2020). Flexible treatment of time-varying covariates with time unstructured data. Structural Equation Modeling, 27(2), 298-317. https://doi.org/10.1080/10705511.2019.1627213

McNeish, D. M., \& Stapleton, L. M. (2016). The effect of small sample size on two-level model estimates: A review and illustration. Educucational Psychology Review, 28, 295-314. https://doi.org/10.1007/s10648-014-9287-x

Muthén, L. K. \& Muthén, B. O. (1998-2017). Mplus user’s guide. ( $8^{\text {th }}$ ed.). Muthén \& Muthén. Preacher, K. J., Zhang, Z., \& Zyphur, M. J. (2011). Alternative methods for assessing mediation in multilevel data: The advantages of multilevel SEM. Structural Equation Modeling, 18(2), 161-182. https://doi.org/10.1080/10705511.2011.557329

Preacher, K. J., Zhang, Z., \& Zyphur, M. J. (2016). Multilevel structural equation models for assessing moderation within and across levels of analysis. Psychological Methods, 21(2), 189-205. https://doi.org/10.1037/met0000052

Raudenbush, S., \& Bryk, A. (2002). Hierarchical linear models (2nd ed.). Sage.

Robitaille, A., Piccinin, A. M., Muniz-Terrera, G., Hoffman, L., Johansson, B., Deeg, D. J. H., Aartsen, M. J., Comijs, H. C., \& Hofer, S. M. (2013). Longitudinal mediation of processing speed on age-related change in memory and fluid intelligence. Psychology and Aging, 28(4), 887-901. https://doi.org/10.1037/a0033316

Scott, P. W. (2020). Accounting for time-varying inter-individual differences in trajectories when assessing cross-lagged models. Structural Equation Modeling. Advanced online publication. https://doi.org/10.1080/10705511.2020.1819815 
Singer, J., \& Willett, J. (2003). Applied longitudinal data analysis: Modeling change and event occurrence. Oxford University Press.

Sliwinski, M., \& Buschke, H. (2004). Modeling intraindividual cognitive change in aging adults: Results from the Einstein aging studies. Aging, Neuropsychology, and Cognition, 11(23), 196-211. https://doi.org/10.1080/13825580490511080

Sliwinski, M. J., Hoffman, L., \& Hofer, S. M. (2010). Evaluating convergence of within-person change and between-person age differences in age-heterogeneous longitudinal studies. Research in Human Development, 7(1), 45-60. https://doi.org/10.1080/15427600903578169

Snijders, T. A. B., \& Bosker, R. J. (2012). Multilevel analysis: An introduction to basic and advanced multilevel modeling (2nd ed.). Sage.

Tuliao A. P., Hoffman L., \& McChargue, D. E. (2017). Measuring individual differences in responses to date-rape vignettes using latent variable models. Aggressive Behavior, 43(1), 60-73. https://doi.org/10.1002/ab.21662

Usami, S., Murayama, K., \& Hamaker, E. L. (2019). A unified framework of longitudinal models to examine reciprocal relations. Psychological Methods, 24(5), 637-657. http://dx.doi.org/10.1037/met0000210

Wang, L. (P.), \& Maxwell, S. E. (2015). On disaggregating between-person and within-person effects with longitudinal data using multilevel models. Psychological Methods, 20(1), 63-83. https://doi.org/10.1037/met0000030

Willett, J. B. (1989). Some results on reliability for the longitudinal measurement of change: Implications for the design of studies of individual growth. Educational \& Psychological Measurement, 49, 587-602. https://psycnet.apa.org/doi/10.1177/001316448904900309 
Zyphur, M. J., Allison, P. D., Tay, L., Voelkle, M. C., Preacher, K. J., Zhang, Z., Hamaker, E. L., Shamsollahi, A., Pierides, D. C., Koval, P., \& Diener, E. (2020). From data to causes I: Building a general cross-lagged panel model (GCLM). Organizational Research Methods, 23(4), 651-687. https://doi.org/10.1177\%2F1094428119847278

Zyphur, M. J., Voelkle, M. C., Tay, L., Allison, P. D., Preacher, K. J., Zhang, Z., Hamaker, E. L., Shamsollahi, A., Pierides, D. C., Koval, P., \& Diener, E. (2020). From data to causes II: Comparing approaches to panel data analysis. Organizational Research Methods, 23(4), 688-716. https://doi.org/10.1177\%2F1094428119847280 


\section{Table 1}

Results for Motivating Example Data of Time-Varying Speed Predicting Time-Varying Memory

\begin{tabular}{|c|c|c|c|c|c|c|}
\hline \multirow{2}{*}{$\begin{array}{l}\text { Models with Concurrent WP } \\
\text { Fixed Effects without a } \\
\text { Speed Time Slope Predictor }\end{array}$} & \multicolumn{2}{|c|}{ Equation 3 (PMC) } & \multicolumn{2}{|c|}{ Equation 3 (BLC) } & \multicolumn{2}{|c|}{ Equation 4} \\
\hline & EST & $\mathrm{SE}$ & EST & $\mathrm{SE}$ & EST & $\mathrm{SE}$ \\
\hline $\begin{array}{l}\text { Speed Residual Predicting } \\
\text { Memory Residual }\end{array}$ & 0.571 & 0.096 & 0.821 & 0.092 & 0.571 & 0.010 \\
\hline $\begin{array}{l}\text { Speed Intercept Predicting } \\
\text { Memory Intercept }\end{array}$ & 2.223 & 0.140 & 1.940 & 0.132 & 2.341 & 0.150 \\
\hline $\begin{array}{l}\text { Speed Intercept Predicting } \\
\text { Memory Time Slope }\end{array}$ & 0.126 & 0.037 & 0.089 & 0.034 & 0.135 & 0.040 \\
\hline \multirow{2}{*}{$\begin{array}{l}\text { Models with Concurrent WP } \\
\text { Fixed Effects with a } \\
\text { Speed Time Slope Predictor }\end{array}$} & \multicolumn{2}{|c|}{ Equation 7} & \multicolumn{2}{|c|}{ Equation 8} & & \\
\hline & EST & $\mathrm{SE}$ & EST & $\mathrm{SE}$ & & \\
\hline $\begin{array}{l}\text { Speed Residual Predicting } \\
\text { Memory Residual }\end{array}$ & 0.411 & 0.110 & 0.411 & 0.110 & & \\
\hline $\begin{array}{l}\text { Speed Intercept Predicting } \\
\text { Memory Intercept }\end{array}$ & 2.236 & 0.140 & 2.463 & 0.194 & & \\
\hline $\begin{array}{l}\text { Speed Intercept Predicting } \\
\text { Memory Time Slope }\end{array}$ & 0.116 & 0.037 & 0.053 & 0.065 & & \\
\hline $\begin{array}{l}\text { Speed Time Slope Predicting } \\
\text { Memory Intercept }\end{array}$ & 2.736 & 0.752 & -1.788 & 4.843 & & \\
\hline $\begin{array}{l}\text { Speed Time Slope Predicting } \\
\text { Memory Time Slope }\end{array}$ & 1.296 & 0.198 & 4.419 & 1.867 & & \\
\hline \multirow{2}{*}{$\begin{array}{l}\text { Models with Cross-Lagged } \\
\text { WP Fixed Effects }\end{array}$} & \multicolumn{2}{|c|}{ Equation 5} & \multicolumn{2}{|c|}{ Equation 6} & \multicolumn{2}{|c|}{ Equation 9} \\
\hline & EST & $\mathrm{SE}$ & EST & SE & EST & $\mathrm{SE}$ \\
\hline $\begin{array}{l}\text { Auto-Regressive } \\
\text { Speed }\end{array}$ & 0.683 & 0.018 & 0.061 & 0.034 & 0.028 & 0.044 \\
\hline $\begin{array}{l}\text { Auto-Regressive } \\
\text { Memory }\end{array}$ & 0.614 & 0.020 & 0.102 & 0.034 & 0.055 & 0.045 \\
\hline Cross-Lagged Speed & & & & & & \\
\hline $\begin{array}{l}\text { Predicting } \\
\text { Memory }\end{array}$ & 0.777 & 0.073 & 0.132 & 0.126 & 0.005 & 0.152 \\
\hline $\begin{array}{l}\text { Cross-Lagged Memory } \\
\text { Predicting Speed }\end{array}$ & 0.044 & 0.005 & 0.016 & 0.007 & 0.008 & 0.009 \\
\hline
\end{tabular}

Note. $\mathrm{EST}=$ estimate, $\mathrm{SE}=$ standard error, $\mathrm{WP}=$ within person, $\mathrm{PMC}=$ person-mean-centering, $\mathrm{BLC}=$ baseline-centering. Bold values indicate significant effects at $p<.05$ using a Wald test. 


\section{Figure 1}

An Unconditional Univariate Longitudinal Model for Change over Time as a Structural Equation Model

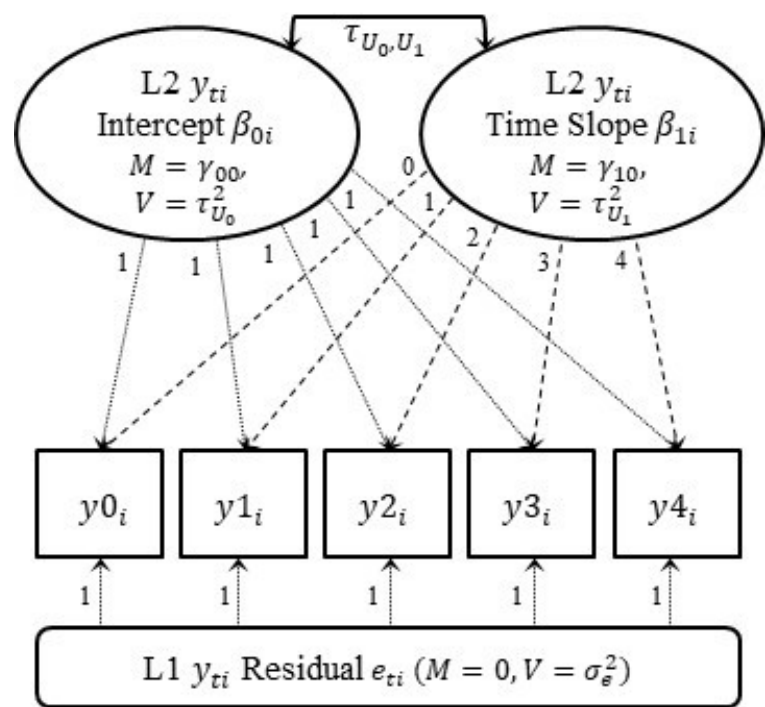

Note. $M=$ mean, $V=$ variance, $\mathrm{L} 1=$ level-1, L2 = level-2. Parameters are from Equation 1 . Rectangles indicate observed variables, ovals indicate latent variables, and the rounded rectangle summarizes the separate residuals for each observed variable whose variances are constrained equal over occasions. Dashed directed arrows indicate constrained regressions, solid directed arrows indicate estimated regressions, and solid undirected arrows indicate covariances. 


\section{Figure 2}

A Structural Equation Model for the Nä̈ve Inclusion of a Time-Varying Predictor

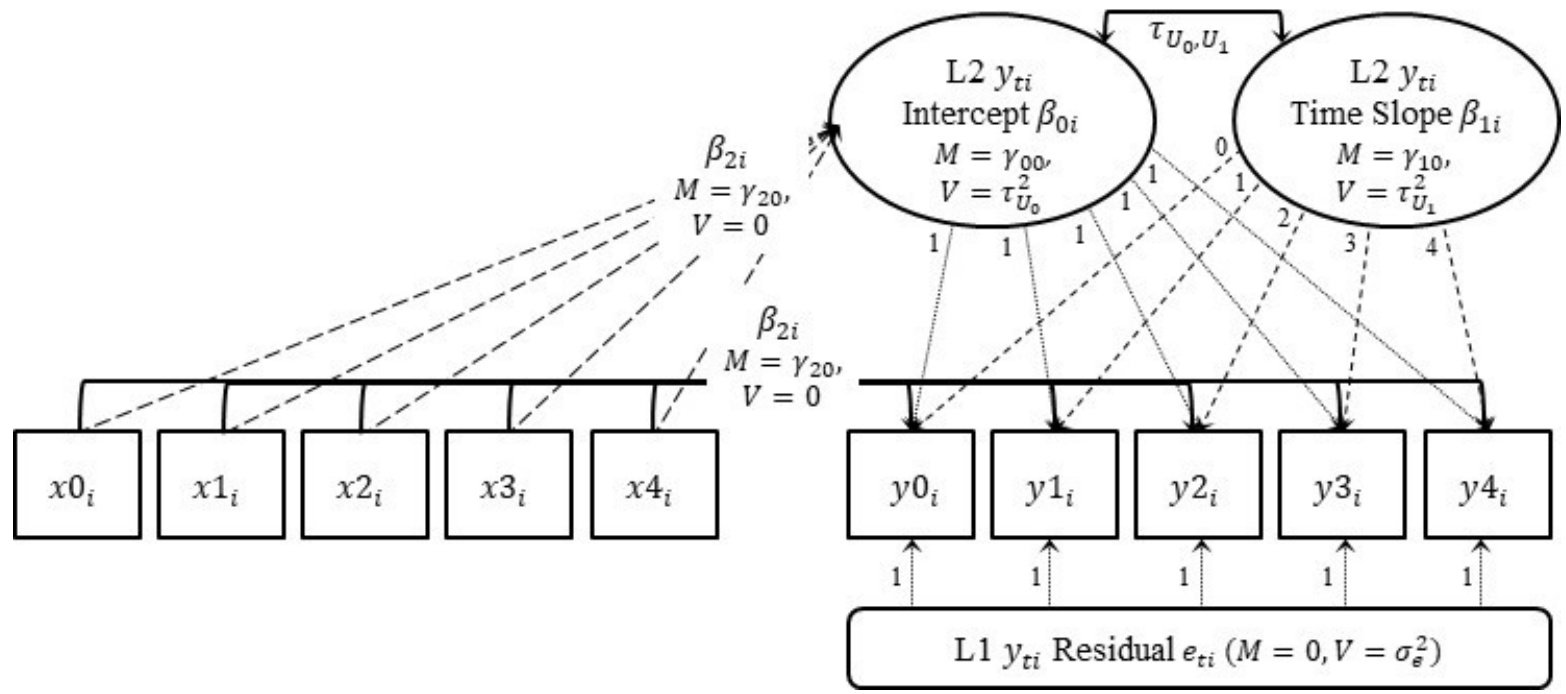

Note. $M=$ mean, $V=$ variance, $\mathrm{L} 1=$ level-1, L2 = level-2. Parameters are from Equation 2. Rectangles indicate observed variables, ovals indicate latent variables, and the rounded rectangle summarizes the separate residuals for each observed variable whose variances are constrained equal over occasions. Dashed directed arrows indicate constrained regressions, solid directed arrows indicate estimated regressions, and solid undirected arrows indicate covariances. The label $\beta_{2 i}$ applies to all regressions of the $x_{i}$ observed variables predicting the $y_{i}$ outcomes at the same occasion (a concurrent effect). The long-dashed arrows are not formally included in the model but are used to schematically represent the implied effect of $\beta_{2 i}$ predicting the L2 random intercept (the between-person intercept effect that is conflated with the within-person residual effect). 


\section{Figure 3}

Structural Equation Models with Between-Person Intercept and Within-Person Residual Associations of a Time-Varying Predictor

(a) Equation 3

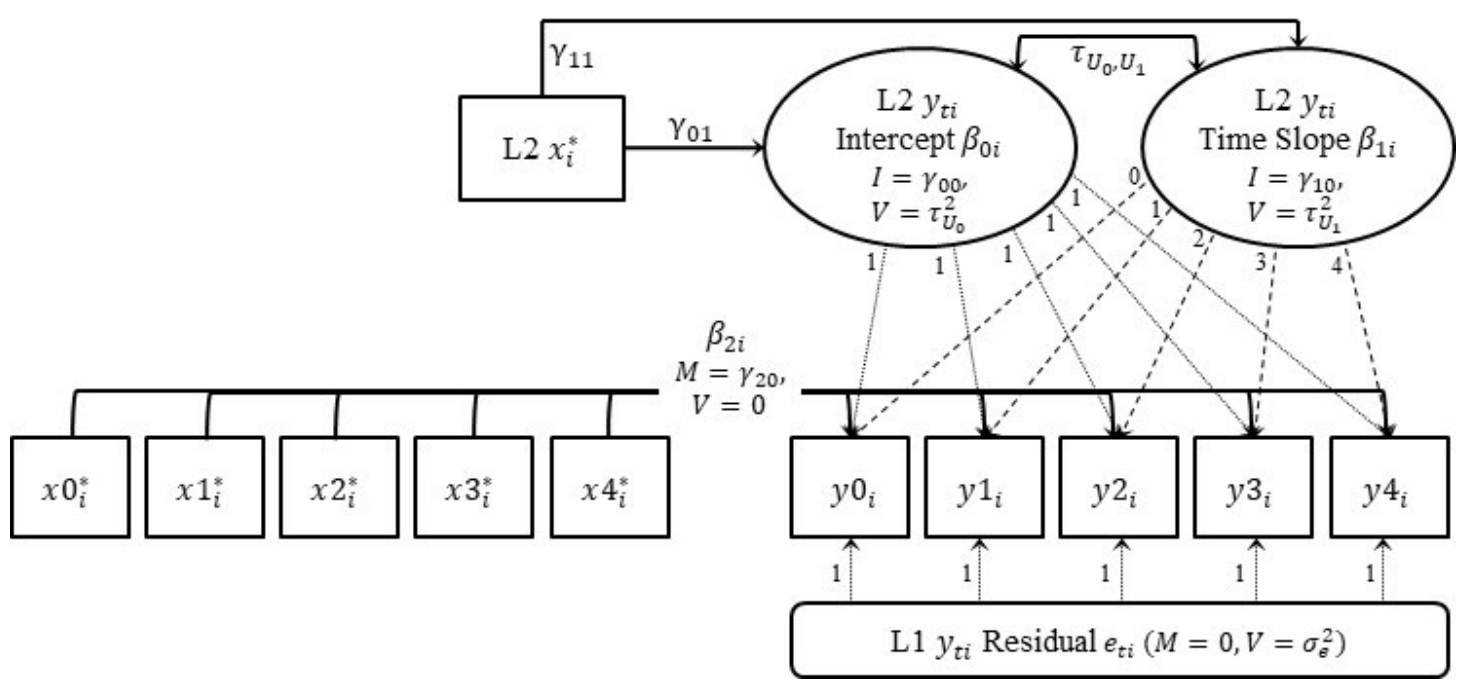

(b) Equation 4

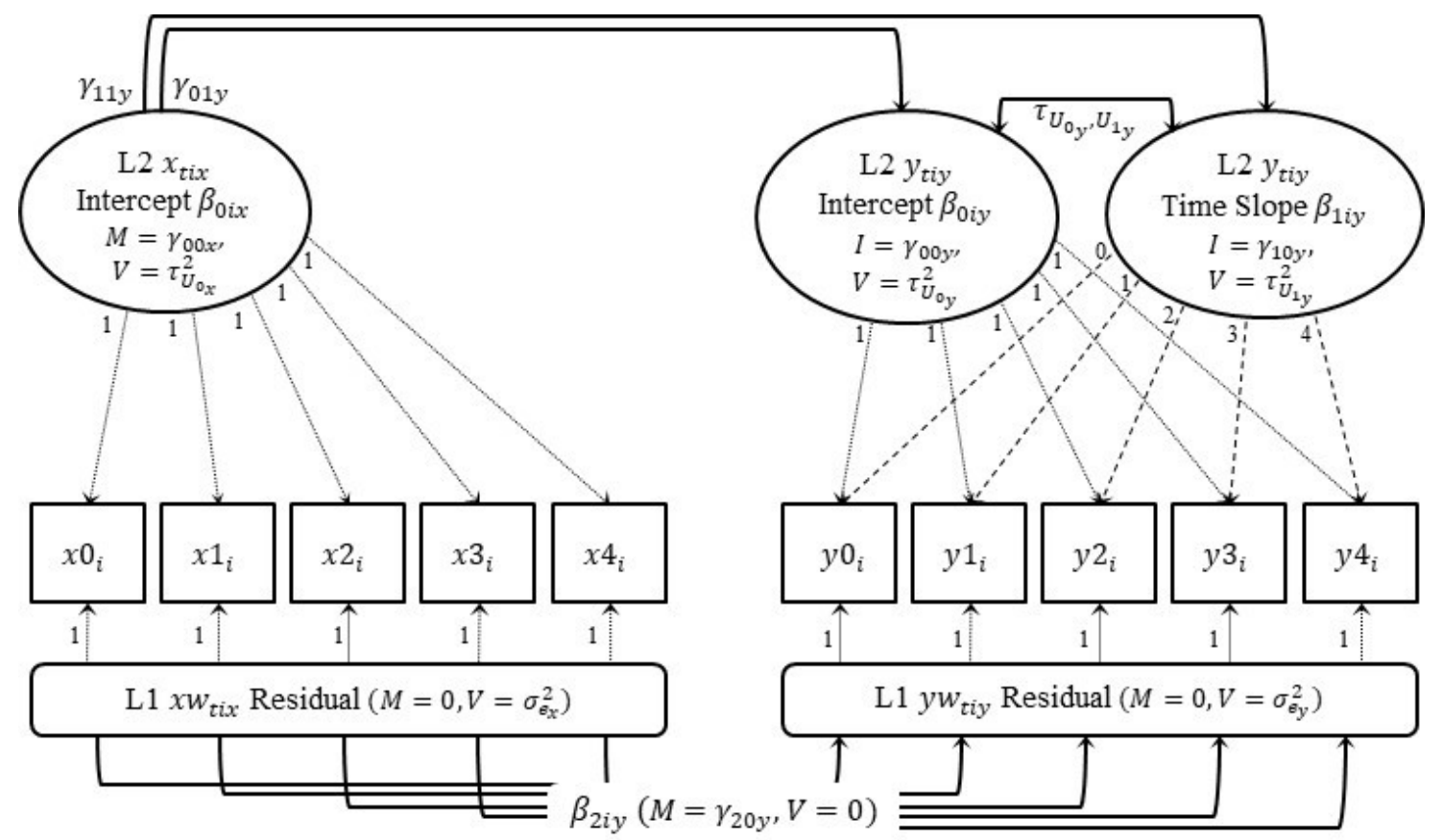

Note. $I=$ intercept, $M=$ mean, $V=$ variance, $\mathrm{L} 1=$ level-1, L2 = level-2. Panel (a) depicts the univariate longitudinal model in Equation 3 and panel (b) depicts the multivariate longitudinal model in Equation 4. Rectangles indicate observed variables, ovals indicate latent variables, and rounded rectangles summarize the separate residuals for each observed variable whose variances are constrained equal over occasions. Dashed directed arrows indicate constrained effects, solid directed arrows indicate estimated effects, and solid undirected arrows indicate covariances. 


\section{Figure 4}

Structural Equation Model Variants of Auto-Regressive Cross-Lag Panel Models

(a) Equation 5

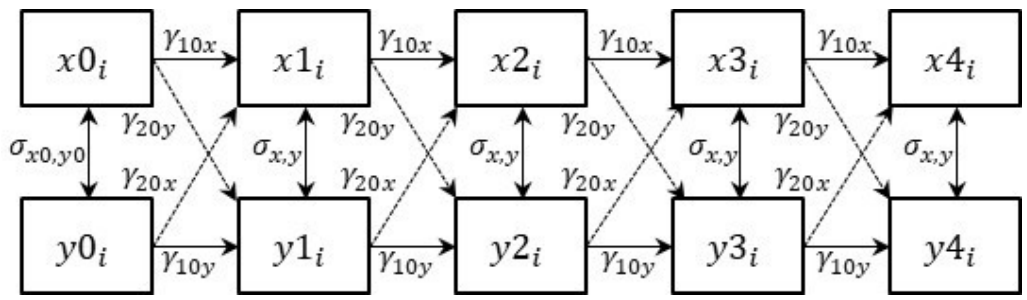

(b) Equation 6

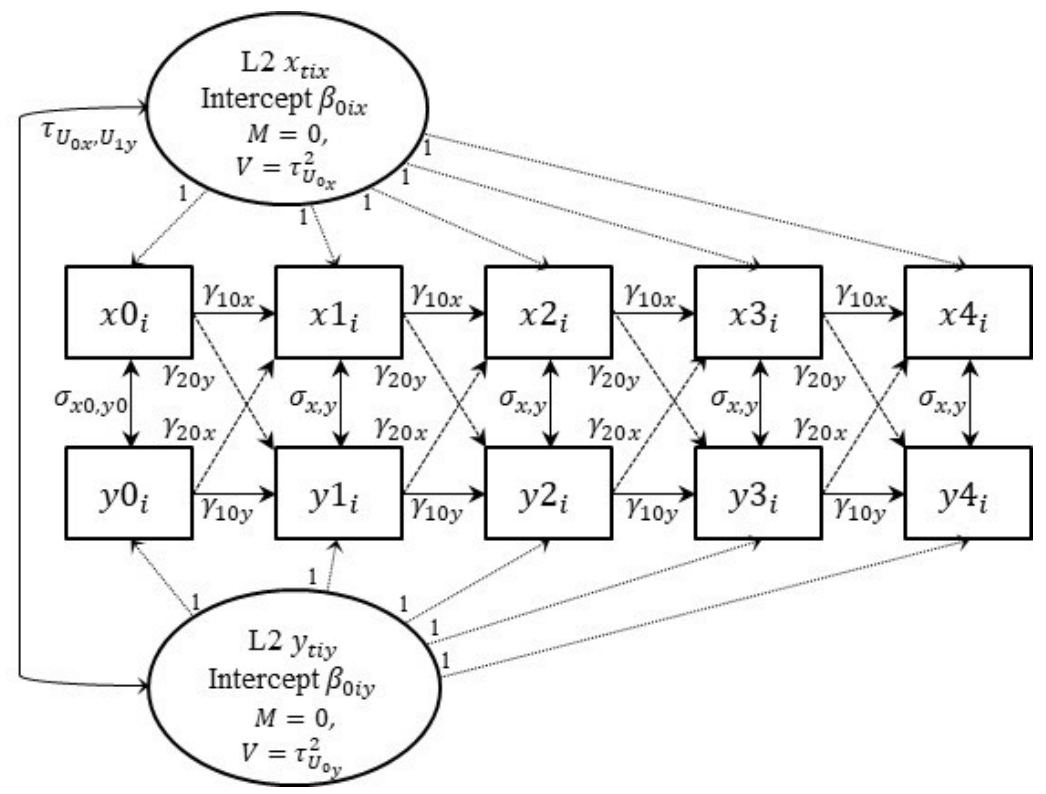

(c) Equation 9

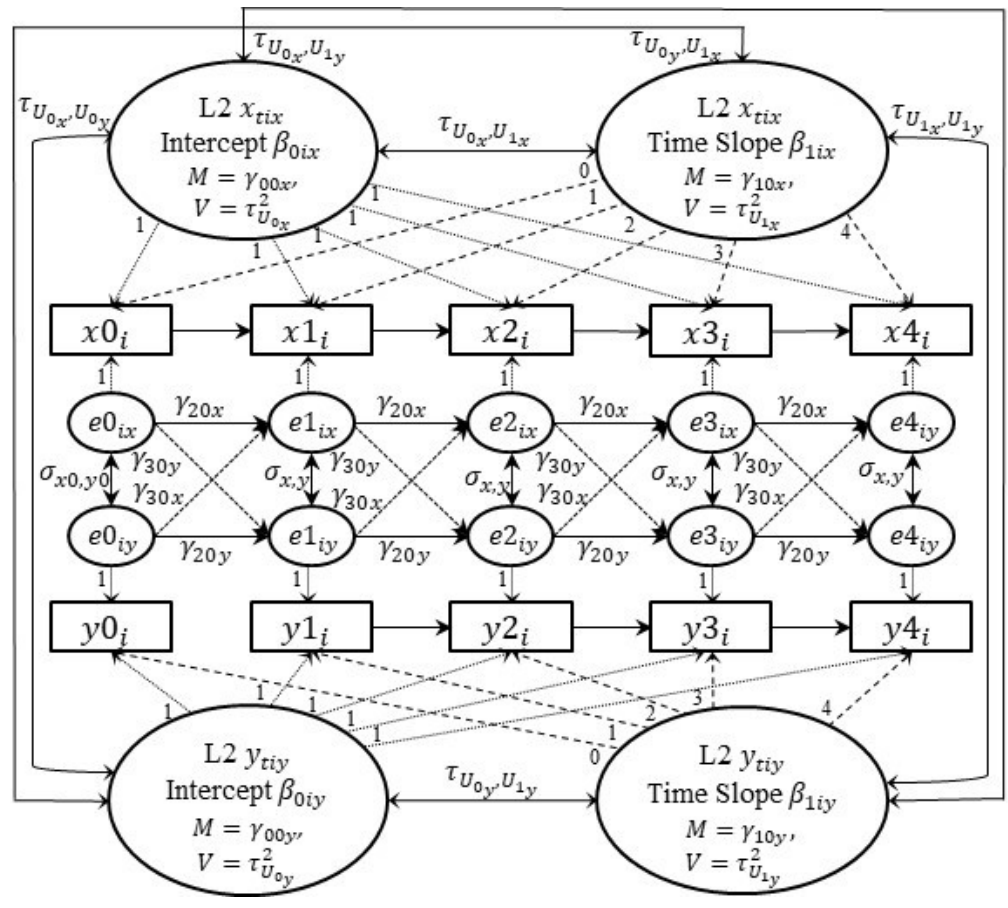


Note. $I=$ intercept, $M=$ mean, $V=$ variance, $\mathrm{L} 1=$ level-1, L2 = level-2. Panel (a) depicts the auto-regressive cross-lag panel model (CPLM) in Equation 5, panel (b) depicts the addition of random intercepts in Equation 6, and panel (c) depicts the addition of random time slopes in Equation 9. Rectangles indicate observed variables and ovals indicate latent variables. Dashed directed arrows indicate constrained effects, solid directed arrows indicate estimated effects, and solid undirected arrows indicate covariances. 


\section{Figure 5}

Structural Equation Models with Between-Person Intercept Effects, Between-Person Time Slope Effects, and Concurrent Within-Person Residual Effect of a Time-Varying Predictor

(a) Equation 7

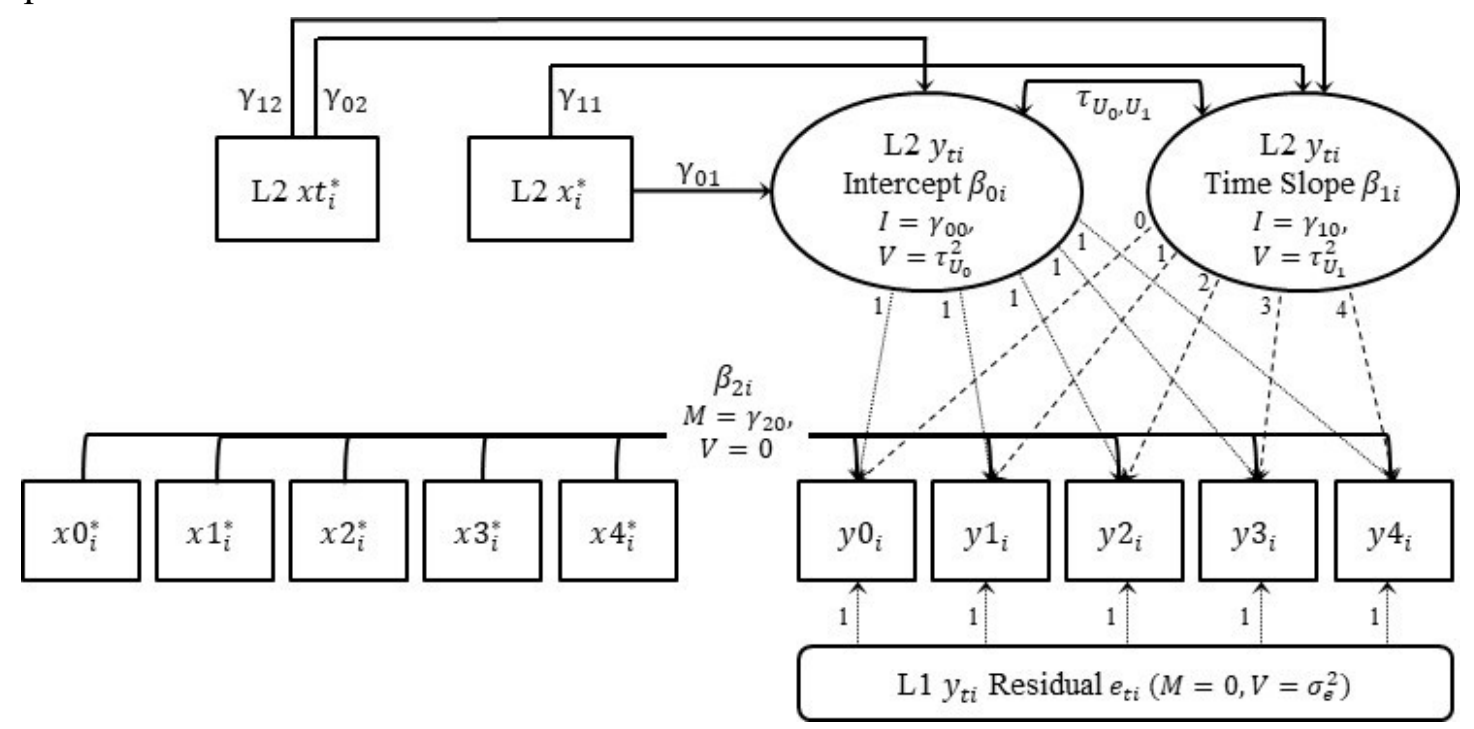

(b) Equation 8

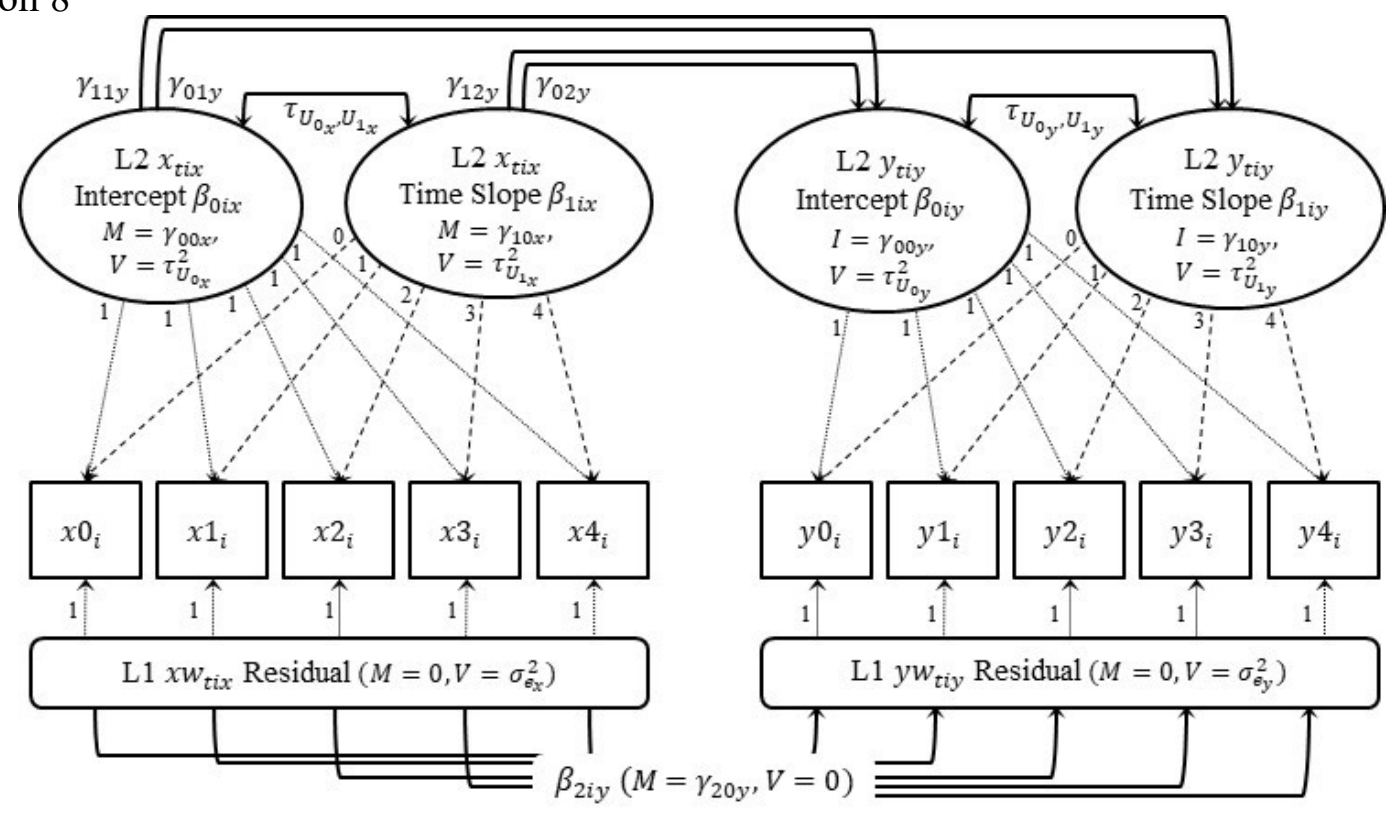

Note. $I=$ intercept, $M=$ mean, $V=$ variance, $\mathrm{L} 1=$ level-1, L2 = level-2. Panel (a) depicts the univariate longitudinal model from Equation 7 and panel (b) depicts the multivariate longitudinal model from Equation 8. Rectangles indicate observed variables, ovals indicate latent variables, and rounded rectangles summarize the separate residuals for each observed variable whose variances are constrained equal over occasions. Dashed directed arrows indicate constrained effects, solid directed arrows indicate estimated effects, and solid undirected arrows indicate covariances. 


\section{Figure 6}

Simulation Results for Models Including Only Directed Between-Person Intercept Effects and Concurrent Within-Person Residual Effects of a Time-Varying Predictor

Model for X Predictor

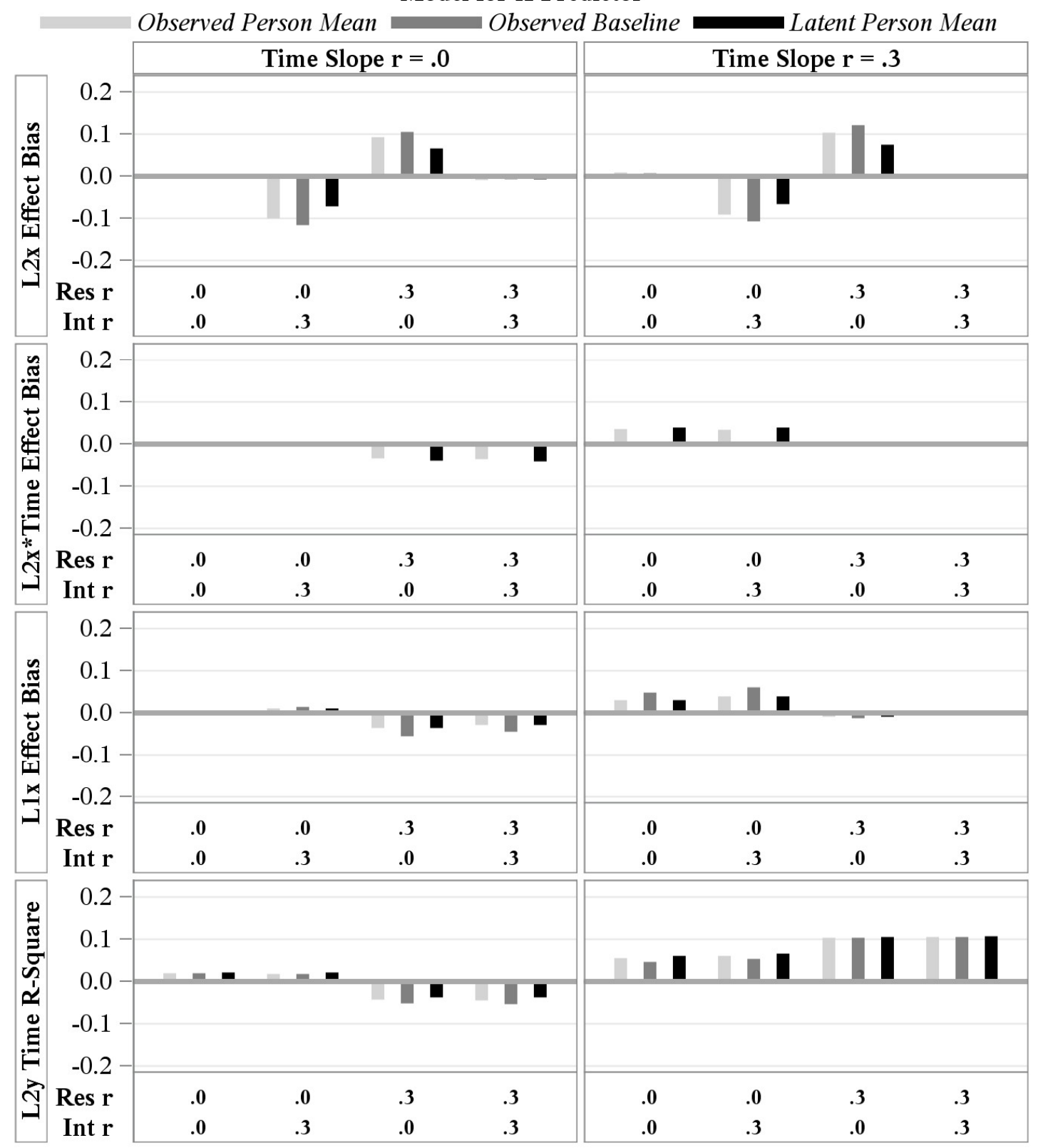

Note . Int $=$ Intercept, Res $=$ Residual, $\mathrm{r}=$ true correlation, $\mathrm{L} 1=$ level-1, $\mathrm{L} 2=$ level-2, $\mathrm{x}=x_{\text {tix }}$ predictor, $\mathrm{y}=y_{t i y}$ outcome. The first three rows show bias in the fixed effects $\gamma_{01 y}, \gamma_{11 y}$, and $\gamma_{10 y}$, respectively, while the fourth row shows pseudo- $R_{U_{1}{ }_{y}}^{2}$ for the time slope variance in $y_{\text {tiy }}$. The labels Observed Person Mean and Observed Baseline refer to the model in Equation 3 (with different predictors), and the label Latent Person Mean refers to the model in Equation 4. 


\section{Figure 7}

Simulation Results for Models Including Directed Between-Person Intercept and Time Slope Effects, as well as Concurrent Within-Person Residual Effects of a Time-Varying Predictor

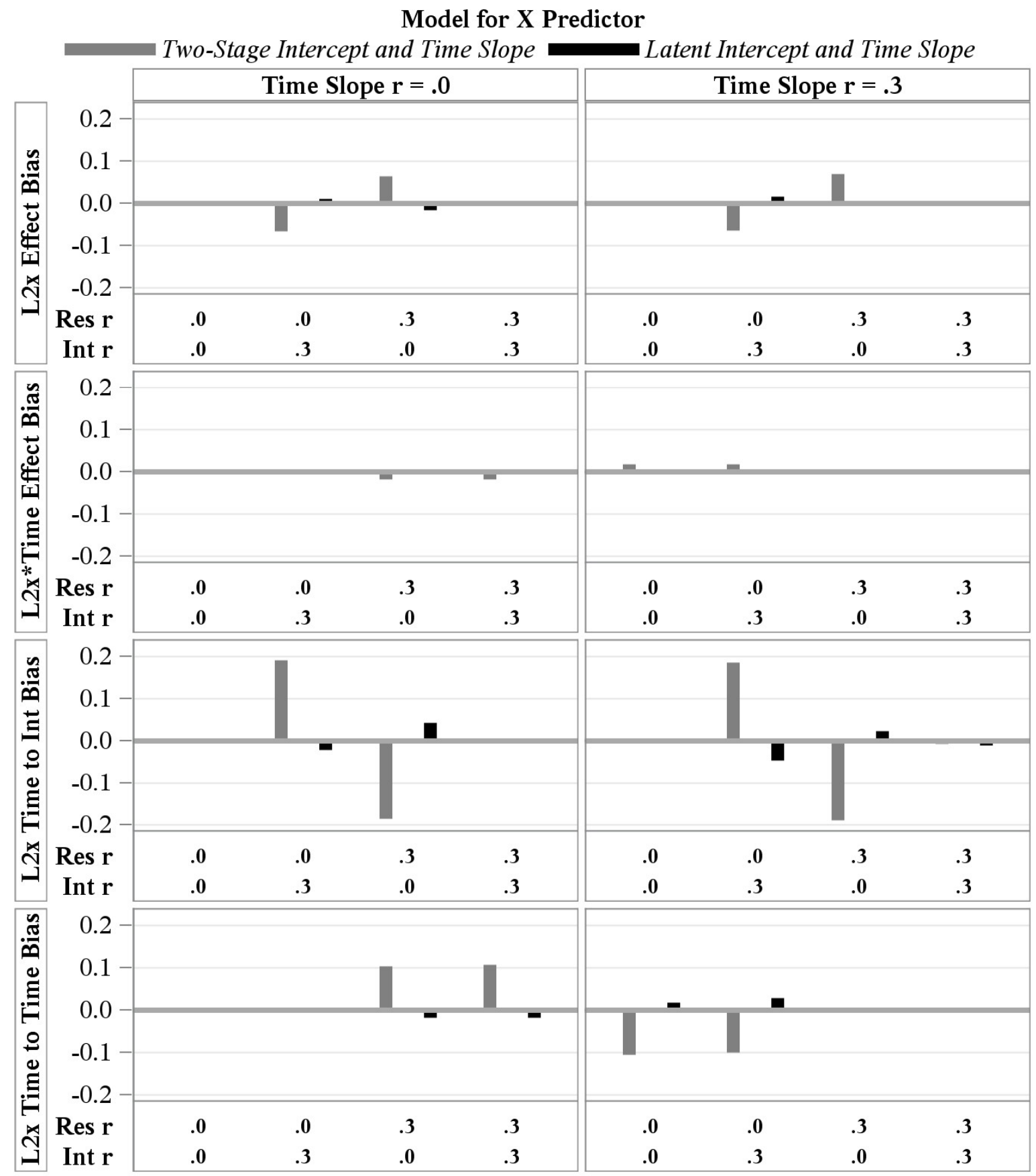

Note. Int $=$ Intercept, Res $=$ Residual, $\mathrm{r}=$ true correlation, $\mathrm{L} 1=$ level-1, $\mathrm{L} 2=$ level-2, $\mathrm{x}=x_{\text {tix }}$ predictor, $\mathrm{y}=y_{\text {tiy }}$ outcome. Rows $1-4$ show bias in the fixed effects $\gamma_{01 y}, \gamma_{11 y}, \gamma_{02 y}$, and $\gamma_{12 y}$, respectively. The label Two-Stage Intercept and Time Slope refers to the model in Equation 7, and the label Latent Intercept and Time Slope refers to the model in Equation 8. 


\section{Figure 8}

Simulation Results for Models with Auto-Regressive and Cross-Lagged Level-1 Fixed Effects

Model for X Predictor

$A R$ with Random Intercept AR with Random Intercept and Time Slope : U1 Random Intercept Uu! Random Intercept and Time Slope

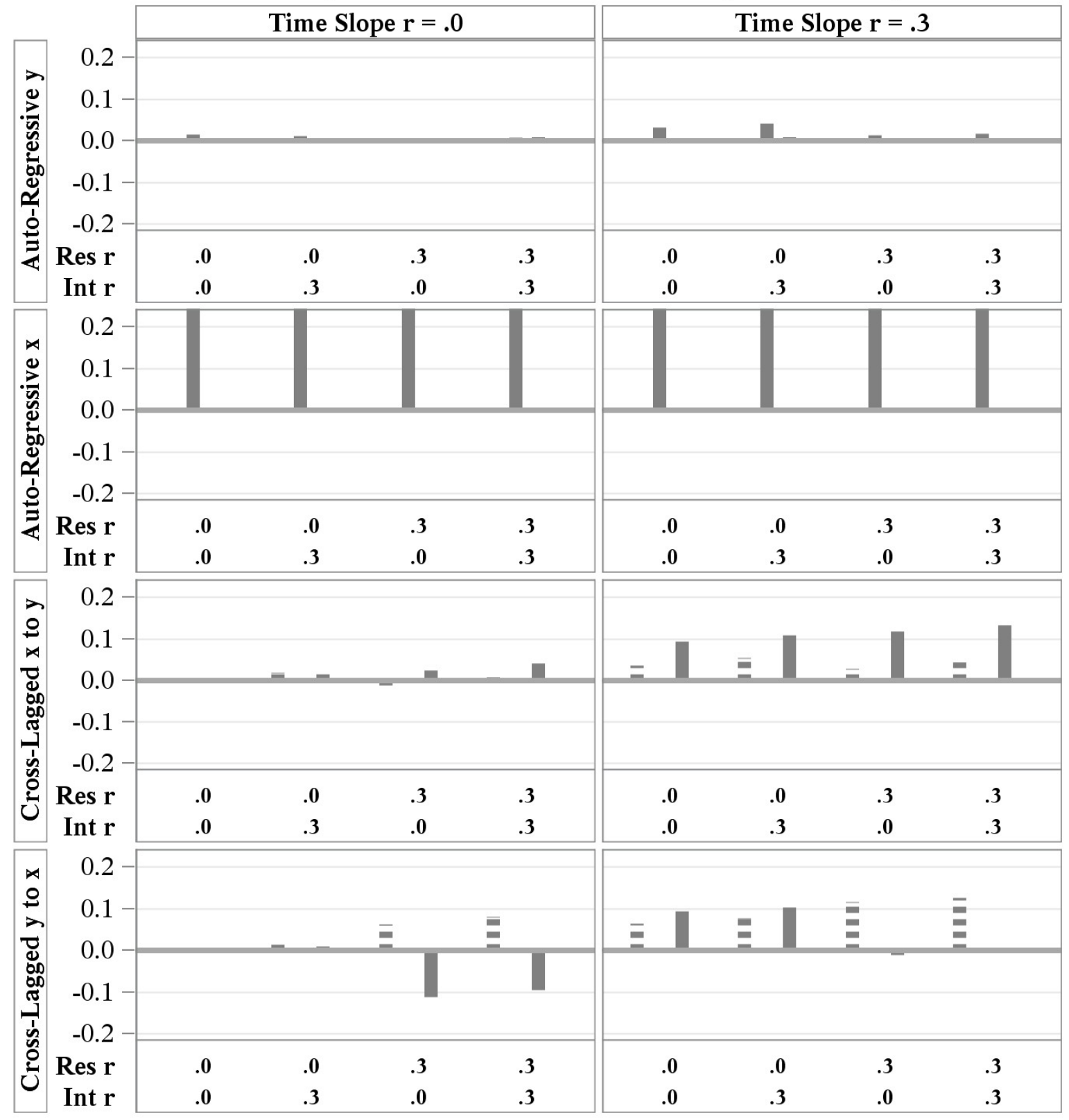

Note . Int $=$ Intercept, Res $=$ Residual, $\mathrm{AR}=$ auto-regressive, $\mathrm{r}=$ true correlation, $\mathrm{x}=x_{\text {tix }}$ predictor, $\mathrm{y}=y_{\text {tiy }}$ outcome. All auto-regressive and cross-lagged level-1 effects were 0 in the population. Rows $1-4$ show bias in the fixed effects $\gamma_{20 y}, \gamma_{20 x}, \gamma_{30}$, and $\gamma_{30}$, respectively. The label Random Intercept and Time Slope refers to the model in Equation 9; the other three models omitted auto-regressive level-1 effects, the $x_{t i x}$ random time slope, or both. 


\section{Appendix A}

\section{Comparison of Models with Time-Varying Predictors and Outcomes}

\section{Table A1}

Models with Directed Level-2 Fixed Effects and Concurrent Level-1 Effects of a Time-Varying Predictor that Contains Individual Differences in Intercept and Change over Time

\begin{tabular}{|c|c|}
\hline $\begin{array}{l}\text { WP Level-1 Model: } \\
\begin{array}{l}y_{t i}=\beta_{0 i}+\beta_{1 i}\left(\text { Time }_{t i}\right) \\
\qquad+\beta_{2 i}\left(x_{t i}\right)+e_{t i}\end{array} \\
\text { BP Level-2 Model: } \\
\text { Intercept: } \quad \beta_{0 i}=\gamma_{00}+U_{0 i} \\
\text { Time Slope: } \quad \beta_{1 i}=\gamma_{10}+U_{1 i} \\
\text { WP } x_{t i}^{*} \text { Slope: } \beta_{2 i}=\gamma_{20}\end{array}$ & $\begin{array}{l}\text { Equation } 2 \text { with an observed predictor: } \\
\text { A single } \gamma_{20} \text { fixed effect of level- } 1 x_{t i} \text { conflates } \\
\text { three separate effects: the BP effect of its } \\
\text { individual intercepts on the } \beta_{0 i} \text { intercept, the BP } \\
\text { effect of its individual time slopes on the } \beta_{1 i} \text { time } \\
\text { slope, and the WP concurrent effect of its same- } \\
\text { occasion residuals on those of } y_{t i} \text {. }\end{array}$ \\
\hline $\begin{array}{l}\text { WP Level-1 Model: } \\
\begin{aligned} y_{t i}=\beta_{0 i}+\beta_{1 i}\left(\text { Time }_{t i}\right) \\
\quad+\beta_{2 i}\left(x_{t i}^{*}\right)+e_{t i}\end{aligned} \\
\text { BP Level-2 Model: } \\
\text { Intercept: } \quad \beta_{0 i}=\gamma_{00}+\gamma_{01}\left(x_{i}^{*}\right)+U_{0 i} \\
\text { Time Slope: } \beta_{1 i}=\gamma_{10}+\gamma_{11}\left(x_{i}^{*}\right)+U_{1 i} \\
\text { WP } x_{t i}^{*} \text { Slope: } \beta_{2 i}=\gamma_{20}\end{array}$ & $\begin{array}{l}\text { Equation } 3 \text { with observed predictors } \\
\text { (created using PMC or BLC): } \\
\text { The } \gamma_{20} \text { level-1 effect of } x_{t i}^{*} \text { (a time-smushed } \\
\text { effect) conflates the BP effect of its individual } \\
\text { time slopes on the } \beta_{1 i} \text { time slope and the WP } \\
\text { concurrent effect of its same-occasion residuals } \\
\text { on those of } y_{t i} \text {. The level-2 effect of } x_{i}^{*} \text { on the } \beta_{0 i} \\
\text { intercept will be biased by the WP concurrent } \\
\text { residual effect. When using PMC, } x_{i}^{*} \text { will reflect } \\
\text { intercept differences at the mean occasion; when } \\
\text { using BLC, } x_{i}^{*} \text { will reflect both intercept } \\
\text { differences and the WP residual at Time } e_{t i}=0 \text {. }\end{array}$ \\
\hline $\begin{array}{l}\text { WP Level-1 Model: } \\
\begin{aligned} x_{t i x} & =\beta_{0 i x}+x w_{t i x} \\
x w_{t i x} & =e_{t i x} \\
y_{t i y} & =\beta_{0 i y}+\beta_{1 \mathrm{i} y}\left(\text { Time }_{t i}\right)+y w_{t i y} \\
y w_{t i y} & =\beta_{2 i y}\left(x w_{t i x}\right)+e_{t i y}\end{aligned} \\
\text { BP Level-2 Model: } \\
x_{t i x} \text { Intercept: } \\
\quad \beta_{0 i x}=\gamma_{00 x}+U_{0 i x} \\
y_{t i y} \text { Intercept: } \\
\quad \beta_{0 i y}=\gamma_{00 y}+\gamma_{01 y}\left(\beta_{0 i x}\right)+U_{0 i y} \\
y_{t i y} \text { Time Slope: } \\
\beta_{1 i y}=\gamma_{10 y}+\gamma_{11 y}\left(\beta_{0 i x}\right)+U_{1 i y} \\
y w_{t i y} \text { WP } x w_{t i x} \text { Slope: } \beta_{2 i y}=\gamma_{20 y}\end{array}$ & $\begin{array}{l}\text { Equation } 4 \text { with latent predictors (created using a } \\
\text { multivariate model predicting } x_{\text {tix }} \text { and } y_{t i y} \text { ): } \\
\text { The } \gamma_{20 y} \text { level-1 effect of } x w_{\text {tix }} \text { (a time-smushed } \\
\text { effect) conflates the BP effect of its individual } \\
\text { time slopes on the } \beta_{1 i y} \text { time slope and the WP } \\
\text { concurrent effect of its same-occasion residuals } \\
\text { on those of } y_{\text {tiy }} \text {. The level-2 effect of } \beta_{0 i x} \text { on the } \\
\beta_{0 i y} \text { intercept will be biased towards the WP } \\
\text { concurrent residual effect. } \beta_{0 i x} \text { will reflect } \\
\text { intercept differences at the mean occasion. }\end{array}$ \\
\hline
\end{tabular}


Table A1, continued

\begin{tabular}{|c|c|}
\hline $\begin{array}{l}\text { WP Level-1 Model: } \\
y_{t i}=\beta_{0 i}+\beta_{1 i}\left(\text { Time }_{t i}\right)+\beta_{2 i}\left(x_{t i}^{*}\right)+e_{t i} \\
\text { BP Level-2 Model: } \\
\text { Intercept: } \quad \beta_{0 i}=\gamma_{00}+\gamma_{01}\left(x_{i}^{*}\right)+\gamma_{02}\left(x t_{i}^{*}\right)+U_{0 i} \\
\text { Time Slope: } \beta_{1 i}=\gamma_{10}+\gamma_{11}\left(x_{i}^{*}\right)+\gamma_{12}\left(x t_{i}^{*}\right)+U_{1 i} \\
\text { WP } x_{t i}^{*} \text { Slope: } \beta_{2 i}=\gamma_{20}\end{array}$ & $\begin{array}{l}\text { Equation } 7 \text { with observed predictors } \\
\text { (created using per-person regressions of } \\
\text { Time } e_{t i} \text { predicting } x_{t i} \text { ): } \\
\text { The } \gamma_{20} \text { level- } 1 \text { effect of } x_{t i}^{*} \text { will } \\
\text { accurately capture the concurrent WP } \\
\text { effect of its same-occasion residuals on } \\
\text { those of } y_{t i} \text {, but the level-2 effects will } \\
\text { not be accurate (as indicated by the bias } \\
\text { shown for the gray bars in Figure } 7 \text { ). }\end{array}$ \\
\hline 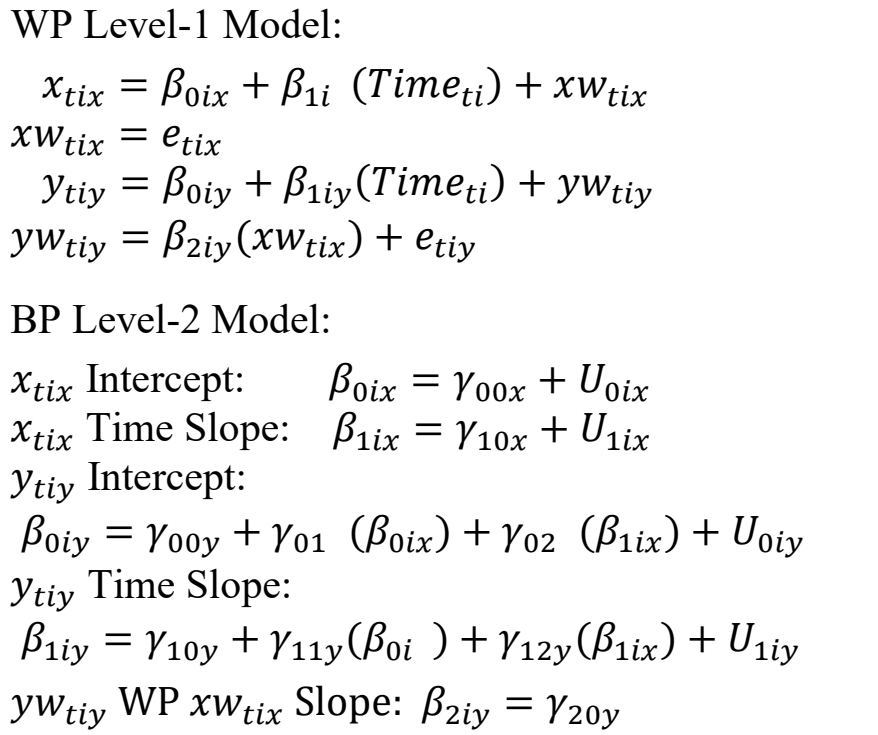 & $\begin{array}{l}\text { Equation } 8 \text { with latent predictors } \\
\text { (created using a multivariate model } \\
\text { predicting } x_{t i x} \text { and } y_{\text {tiy }} \text { ): } \\
\text { All effects will be accurately captured. }\end{array}$ \\
\hline
\end{tabular}




\section{Table A2}

Models with Undirected Covariances (and Cross-Lagged Level-1 Effects) of a Time-Varying Predictor that Contains Individual Differences in Change over Time

\begin{tabular}{|c|c|}
\hline $\begin{array}{l}\text { Single-Level Model: } \\
\begin{aligned} x_{t i x}=\gamma_{t 0 x}+ & \gamma_{10 x}\left(x_{t-1 i}\right)+\gamma_{20 x}\left(y_{t-1 i}\right) \\
& +e_{t i x} \\
y_{t i y}=\gamma_{t 0 y}+ & \gamma_{10 y}\left(y_{t-1 i}\right)+\gamma_{20 y}\left(x_{t-1 i}\right) \\
& +e_{t i y}\end{aligned}\end{array}$ & $\begin{array}{l}\text { Equation } 5 \text { with observed predictors: } \\
\text { The single } \gamma_{20} \text { fixed effect of each variable } \\
\text { conflates three separate effects on the other } \\
\text { variable: the BP effect of its individual } \\
\text { intercepts on the other's intercept, the BP } \\
\text { effect of its individual time slopes on the } \\
\text { other's time slope, and the WP lagged effect } \\
\text { of its residuals on those of the other variable } \\
\text { at the subsequent occasion. }\end{array}$ \\
\hline 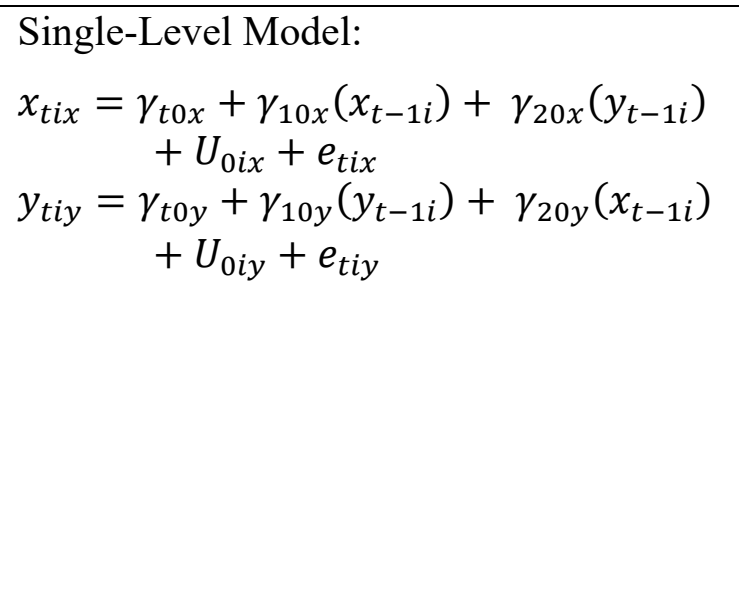 & $\begin{array}{l}\text { Equation } 6 \text { with observed or latent } \\
\text { level-1 predictors: } \\
\text { The } \gamma_{20} \text { fixed effect of each variable (a time- } \\
\text { smushed effect) conflates two separate } \\
\text { effects on the other variable: the BP effect of } \\
\text { its individual time slopes on the other's time } \\
\text { slope, and the WP lagged effect of its } \\
\text { residuals on those of the other variable at the } \\
\text { subsequent occasion. The BP intercept effect } \\
\text { of one variable on the other at the mean } \\
\text { occasion is captured by the intercept } \\
\text { covariance instead. }\end{array}$ \\
\hline $\begin{array}{l}\text { WP Level-1 Model: } \\
\begin{aligned} x+i x & =\beta_{0 i x}+\beta_{1 i}\left(\text { Time }_{t i}\right)+x w_{t i x} \\
x w_{t i x} & =\beta_{2 i x}\left(x_{t-1 i}\right)+\beta_{3 i x}\left(y_{t-1 i}\right)+e_{t i x} \\
y_{t i y} & =\beta_{0 i y}+\beta_{1 i y}\left(\text { Time }_{t i}\right)+y w_{t i y} \\
y w_{t i y} & =\beta_{2 i y}\left(y_{t-1 i}\right)+\beta_{3 i y}\left(x_{t-1 i}\right)+e_{t i y}\end{aligned} \\
\text { BP Level-2 Model and Level-2 Matrix G: } \\
x_{t i x} \text { Intercept: } \quad \beta_{0 i x}=\gamma_{00 x}+U_{0 i} \\
x_{t i x} \text { Time Slope: } \beta_{1 i x}=\gamma_{10}+U_{1 i x} \\
y_{t i y} \text { Intercept: } \quad \beta_{0 i y}=\gamma_{00}+U_{0 i y} \\
y_{t i y} \text { Time Slope: } \beta_{1 i y}=\gamma_{10 y}+U_{1 i y} \\
x w_{t i x} \text { WP AR } x w_{t i x} \text { Slope: } \beta_{2 i x}=\gamma_{20 x} \\
x w_{t i x} \text { WP CL } y w_{t i y} \text { Slope: } \beta_{3 i x}=\gamma_{30 x} \\
y w_{t i y} \text { WP AR } y w_{t i y} \text { Slope: } \beta_{2 i y}=\gamma_{20 y} \\
y w_{t i y} \text { WP CL } x w_{t i x} \text { Slope: } \beta_{3 i y}=\gamma_{30 y}\end{array}$ & $\begin{array}{l}\text { Equation } 9 \text { with latent predictors: } \\
\text { All effects will be accurately captured, } \\
\text { although the relations among the intercepts } \\
\text { and time slopes across variables will be } \\
\text { given as covariances instead of fixed effects. }\end{array}$ \\
\hline
\end{tabular}




\section{Appendix B}

\section{Derivation for Conflation of the BP Effect of Individual Time Slopes Predicting the}

\section{Outcome Time Slopes with the WP Concurrent Effect of Time-Specific Residuals}

The directed effects model from Equation 8 including individual intercepts and individual time slopes for both $x_{t i x}$ and $y_{t i y}$ variables, as well the relations between them, is shown in composite form (with separate lines for the effects of each $x_{t i x}$ predictor) in Equation B1:

$$
\begin{aligned}
x_{t i x}=[ & \left.\gamma_{00 x}+U_{0 i x}\right]+\left[\gamma_{10 x}+U_{1 i x}\right]\left(\text { Time }_{t i}\right)+e_{t i x} \\
y_{t i y}=[ & \left.\gamma_{00 y}+U_{0 i y}\right]+\left[\gamma_{10 y}+U_{1 i x}\right]\left(\text { Time }_{t i}\right)+e_{t i y} \\
& +\gamma_{20 y}\left(e_{t i x}\right) \\
& +\gamma_{01 y}\left(\gamma_{00 x}+U_{0 i x}\right)+\gamma_{02 y}\left(\gamma_{10 x}+U_{1 i x}\right) \\
& +\gamma_{11 y}\left(\gamma_{00 x}+U_{0 i x}\right)\left(\text { Time }_{t i}\right)+\gamma_{12 y}\left(\gamma_{10 x}+U_{1 i x}\right)\left(\text { Time }_{t i}\right)
\end{aligned}
$$

from which the contents of the observed predictors used in Equation 3 can be created.

\section{Predictor Contents when Using Person-Mean-Centering (PMC)}

Using PMC, the level-2 $x_{i}^{*}$ and level-1 $x_{\text {tix }}^{*}$ predictors can be derived as follows. First, let the level-2 $x_{i}^{*}$ predictor be equal to the individual mean of $x_{t i x}$ over time, as in Equation B2:

$$
\begin{aligned}
x_{i}^{*} & =\frac{\sum_{t=0}^{n-1} x_{t i x}}{n}=\frac{\sum_{t=0}^{T}\left[\left[\gamma_{00 x}+U_{0 i x}\right]+\left[\gamma_{10 x}+U_{1 i x}\right]\left(\text { Time }_{t i}\right)+e_{t i x}\right]}{n} \\
& =\left[\gamma_{00 x}+U_{0 i x}\right]+\left[\gamma_{10 x}+U_{1 i x}\right]\left(\overline{\text { Trme }}_{t i}\right)+\bar{e}_{i x}
\end{aligned}
$$

in which $t$ indicates the value of Time $_{t i}$ for each occasion included in the person mean and $n$ indicates the number of level-1 occasions. Next, the level- $1 x_{\text {tix }}^{*}$ predictor can be created by subtracting the observed person mean just found in Equation B2, as shown in Equation B3:

$$
\begin{aligned}
x_{t i x}^{*}= & x_{t i x}-x_{i}^{*} \\
= & x_{t i x}-\left[\gamma_{00 x}+U_{0 i x}\right]-\left[\gamma_{10 x}+U_{1 i x}\right]\left(\overline{T i m e}_{t i}\right)-\bar{e}_{i x} \\
= & {\left[\gamma_{00 x}+U_{0 i x}\right]+\left[\gamma_{10 x}+U_{1 i x}\right]\left(\text { Time }_{t i}\right)+e_{t i x} } \\
& -\left[\gamma_{00 x}+U_{0 i x}\right]-\left[\gamma_{10 x}+U_{1 i x}\right]\left(\overline{T i m e}_{t i}\right)-\bar{e}_{i x} \\
= & {\left[\gamma_{10 x}+U_{1 i x}\right]\left(\text { Time }_{t i}-\overline{T i m e}_{t i}\right)-\bar{e}_{i x}+e_{t i x} }
\end{aligned}
$$

and the model from Equation 3 using PMC can then be expressed using these expanded versions of the observed predictors as follows in Equation B4: 


$$
\begin{aligned}
y_{t i y}= & {\left[\gamma_{00 y}+U_{0 i y}\right]+\left[\gamma_{10 y}+U_{1 i x}\right]\left(\text { Time }_{t i}\right)+e_{t i y} } \\
& +\gamma_{20 y}\left\{x_{t i x}^{*}\right\} \\
& +\gamma_{01 y}\left\{x_{i}^{*}\right\} \\
& +\gamma_{11 y}\left\{x_{i}^{*}\right\}\left(\text { Time }_{t i y}\right) \\
= & \left.\gamma_{00 y}+U_{0 i y}\right]+\left[\gamma_{10 y}+U_{1 i x}\right]\left(\text { Time }_{t i}\right)+e_{t i y} \\
& +\gamma_{20 y}\left\{\left[\gamma_{10 x}+U_{1 i x}\right]\left(\text { Time }_{t i x}-\overline{T i m e}_{i x}\right)-\bar{e}_{i x}+e_{t i x}\right\} \\
& +\gamma_{01 y}\left\{\left[\gamma_{00 x}+U_{0 i x}\right]+\left[\gamma_{10 x}+U_{1 i x}\right]\left(\overline{T i m e}_{i x}\right)+\bar{e}_{i x}\right\} \\
& +\gamma_{11 y}\left\{\left[\gamma_{00 x}+U_{0 i x}\right]+\left[\gamma_{10 x}+U_{1 i x}\right]\left(\overline{T i m e}_{i x}\right)+\bar{e}_{i x}\right\}\left(\text { Time }_{t i y}\right)
\end{aligned}
$$

in which the conflation of the obtained level-1 and level-2 effects is now shown. The level-1 fixed effect $\gamma_{20 y}$ (predicting the $y_{\text {tiy }}$ outcome) reflects not only the effect of the $x_{\text {tix }}$ level-1 residual $\left[e_{t i x}\right]$ as intended, but also the effect of the $x_{t i x}$ individual time slope on the $y_{t i y}$ time slope $\left[\gamma_{10 x}+U_{1 i x}\right]\left(\right.$ Time $\left._{t i x}-\overline{T ı m e}_{i x}\right)$, minus the effect of the $x_{t i x}$ person mean level-1 residual $\left[\bar{e}_{i x}\right]$. The level-2 fixed effect $\gamma_{01 y}$ (predicting the $y_{\text {tiy }}$ level-2 intercept at Time $_{t i}=0$ ) reflects not only the effect of the $x_{t i x}$ level-2 intercept at Time $_{t i}=0\left[\gamma_{00 x}+U_{0 i}\right]$ as intended, but also the effects of the $x_{t i x}$ individual time slope multiplied by the mean occasion, $\left[\gamma_{10 x}+U_{1 i x}\right]\left(\overline{T r m e}_{i x}\right)$, and the $x_{t i x}$ person mean level-1 residual $\left[\bar{e}_{i x}\right]$. The same problems occur for the level-2 fixed effect $\gamma_{11 y}$ (predicting the $y_{t i y}$ level-2 time slope). Note that the effects of the $x_{t i x}$ person mean level-1 residual $\left[\bar{e}_{i x}\right]$ will be removed when using a latent person mean (as in Equation 4) instead of an observed person mean.

\section{Predictor Contents when Using Baseline-Centering (BLC)}

Using BLC, the level-2 $x_{i}^{*}$ and level- $1 x_{t i x}^{*}$ predictors can be derived as follows. First, let the level-2 $x_{i}^{*}$ predictor be equal to $x_{t i x}$ at Time $_{t i}=0$ as follows in Equation B5:

$$
x_{i}^{*}=\left[\gamma_{00 x}+U_{0 i x}\right]+e_{0 i x}
$$

in which $e_{0 i}$ is the $x_{t i x}$ residual at Time $e_{t i}=0$. Next, the level- $1 x_{t i x}^{*}$ predictor can be created by subtracting the observed baseline value just found in Equation B5, as shown in Equation B6: 


$$
\begin{aligned}
x_{t i x}^{*}= & x_{t i x}-x_{i}^{*} \\
= & x_{t i x}-\left[\gamma_{00 x}+U_{0 i x}\right]-e_{0 i x} \\
= & {\left[\gamma_{00 x}+U_{0 i}\right]+\left[\gamma_{10 x}+U_{1 i}\right]\left(\text { Time }_{t i}\right)+e_{t i x} } \\
& -\left[\gamma_{00 x}+U_{0 i x}\right]-e_{1 i} \\
= & {\left[\gamma_{10 x}+U_{1 i x}\right]\left(\text { Time }_{t i}\right)-e_{0 i x}+e_{t i x} }
\end{aligned}
$$

and the model from Equation 3 using BLC can then be expressed using these expanded versions of the observed predictors as follows in Equation B7:

$$
\begin{aligned}
y_{t i y}= & {\left[\gamma_{00 y}+U_{0 i y}\right]+\left[\gamma_{10 y}+U_{1 i x}\right]\left(\text { Time }_{t i}\right)+e_{t i y} } \\
& +\gamma_{20 y}\left\{x_{t i x}^{*}\right\} \\
& +\gamma_{01 y}\left\{x_{i}^{*}\right\} \\
& +\gamma_{11 y}\left\{x_{i}^{*}\right\}\left(\text { Time }_{t i y}\right) \\
= & {\left[\gamma_{00 y}+U_{0 i y}\right]+\left[\gamma_{10 y}+U_{1 i x}\right]\left(\text { Time }_{t i}\right)+e_{t i y} } \\
& +\gamma_{20 y}\left\{\left[\gamma_{10 x}+U_{1 i x}\right]\left(\text { Time }_{t i x}\right)-e_{0 i x}+e_{t i x}\right\} \\
& +\gamma_{01 y}\left\{\left[\gamma_{00 x}+U_{0 i x}\right]+e_{0 i}\right\} \\
& +\gamma_{11 y}\left\{\left[\gamma_{00 x}+U_{0 i x}\right]+e_{0 i x}\right\}\left(\text { Time }_{t i y}\right)
\end{aligned}
$$

in which the conflation of the obtained level-1 and level-2 effects is shown. The level-1 fixed effect $\gamma_{20 y}$ (predicting the $y_{\text {tiy }}$ outcome) reflects not only the effect of the $x_{\text {tix }}$ level- 1 residual $\left[e_{t i x}\right]$ as intended, but also the effect of the $x_{t i x}$ individual time slope on the $y_{t i y}$ time slope $\left[\gamma_{10 x}+U_{1 i x}\right]\left(\right.$ Time $\left._{t i x}-\overline{\text { Time }}_{i x}\right)$, minus the effect of the $x_{t i x}$ level-1 residual at Time $_{t i}=0$ $\left[e_{0 i x}\right]$. The level-2 fixed effect $\gamma_{01 y}$ (predicting the $y_{t i y}$ level-2 intercept at Time $_{t i}=0$ ) reflects not only the effect of the $x_{t i x}$ level-2 intercept at Time ti $=0\left[\gamma_{00}+U_{0 i x}\right]$ as intended, but also the effect of the $x_{t i x}$ level-1 residual at Time $\left._{t i}=0\right)\left[e_{0 i x}\right]$. The same problems occur for the level-2 fixed effect $\gamma_{11}$ (predicting the $y_{\text {tiy }}$ level-2 time slope). 